\title{
Topological Feedback Entropy and Nonlinear Stabilization
}

\author{
Girish N. Nair, Member, IEEE, Robin J. Evans, Fellow, IEEE, Iven M. Y. Mareels, Fellow, IEEE, and \\ William Moran, Member, IEEE
}

\begin{abstract}
It is well known in the field of dynamical systems that entropy can be defined rigorously for completely deterministic open-loop systems. However, such definitions have found limited application in engineering, unlike Shannon's statistical entropy. In this paper, it is shown that the problem of communication-limited stabilization is related to the concept of topological entropy, introduced by Adler et al. as a measure of the information rate of a continuous map on a compact topological space. Using similar open cover techniques, the notion of topological feedback entropy $(T F E)$ is defined in this paper and proposed as a measure of the inherent rate at which a map on a noncompact topological space with inputs generates stability information. It is then proven that a topological dynamical plant can be stabilized into a compact set if and only if the data rate in the feedback loop exceeds the TFE of the plant on the set. By taking appropriate limits in a metric space, the concept of local TFE (LTFE) is defined at fixed points of the plant, and it is shown that the plant is locally uniformly asymptotically stabilizable to a fixed point if and only if the data rate exceeds the plant LTFE at the fixed point. For continuously differentiable plants in Euclidean space, real Jordan forms and volume partitioning arguments are then used to derive an expression for LTFE in terms of the unstable eigenvalues of the fixed point Jacobian.
\end{abstract}

Index Terms-Communication channels, stabilizability, topological entropy.

\section{INTRODUCTION}

A N IMPORTANT consequence of Shannon's pioneering work on statistical information theory was the development of definitions of entropy for deterministic nonlinear dynamical systems, by Kolmogorov and others. These notions provide a framework within which to rigorously discuss the information generation rate, in bits per second, of purely deterministic maps, either in measure-theoretic or topological terms. However, their impact in communications has been somewhat limited, due to the widespread use in that field of statistical source models which are often more naturally analyzed in terms of Shannon entropy. In contrast, deterministic models are commonly used in control. Until recently though, there has been no real motivation to define the information rate in bits/s of a plant in a control system, since it has almost always been assumed that the available outputs of a plant can

Manuscript received June 4, 2003; revised January 1, 2004. Recommended by Guest Editors P. Antsaklis and J. Baillieul. This work was supported by the Australian Research Council under Grants DP0345044 and DP0210197. The work of G. N. Nair was also supported by Melbourne Early Career Researcher and Melbourne Research Grants.

The authors are with the Department of Electrical and Electronic Engineering, University of Melbourne, Melbourne, VIC 3010, Australia (e-mail: gnair@ee.mu.oz.au).

Digital Object Identifier 10.1109/TAC.2004.834105 be transported to the controller with arbitrarily high digital precision.

However, in many developing application areas such as microelectromechanical systems and decentralized tracking, this assumption no longer holds true. The resources available for communication between sensors and controllers in such areas can be severely limited, due to size or cost. This impinges directly on the feedback control performance that can be achieved, since it implies that the data received by various components is either out-of-date or poor in resolution, if not both. In these situations the communications and control issues are bound together and the analysis of one aspect cannot proceed without consideration of the other. There are many issues which need to be considered in order to fully capture the effects of a nonideal digital communication channel on a control system, e.g., limited data rate, variable or stochastic delays, and transmission errors. In this paper we follow a line of inquiry begun in [1] and continued in [2]-[9], amongst others, and focus on the problem of stabilization with a finite data rate.

Within this perimeter, a fundamental question is how to define the intrinsic rate $h$ at which a given plant generates "stability" information. One possibility is to simply define it as the infimum data rate needed to be able to stabilize the plant, and indeed expressions for the infimum rate are available both for noiseless [3], [10]-[15] and stochastic linear systems. However, the weakness of this approach is that it ties the definition of information rate to the particular structure placed on the coding and control scheme. Despite different formulations and assumptions in all the papers just referred to, the same infimum rate was obtained which furthermore was determined only by the unstable, open-loop eigenvalues of the plant and was independent of all parameters of the coding and control scheme. This strongly suggests that it ought to be possible to define an intrinsic information rate for the plant in a manner which makes no reference to coder, decoder or controller structure. There is an analogy here with source coding [16], [17], which is concerned with determining the smallest data rate at which a stochastic source can be coded, transmitted and "reliably" decoded over a noiseless digital channel. There are different formulations and definitions of "reliability," ranging from variable length codes with no errors to fixed-length block codes with arbitrarily small error rates. Nonetheless, in all cases the smallest possible data rate is equal to the Shannon entropy of the source, independent of external constructs.

This paper is divided into five sections, excluding this Introduction. In Section II, we briefly discuss the open cover techniques used by Adler et al.in defining the topological entropy of a continuous map without inputs on a compact topological 
space. Motivated by the generality possible with such methods, we modify them to deal with plants on (possibly noncompact) topological spaces. This leads to the new concept of topological feedback entropy (TFE), which we propose as a rigorous and completely general measure of the rate at which a plant generates initial state information under certain controllability-like constraints.

In Section III, we formulate the problem of controlling a topological dynamical system with a finite feedback data rate, the basic objective being to keep the state contained in a specified compact region. The first theorem of this paper is presented and proven here. This states that the infimum data rate for which the objective is possible is precisely equal to the TFE of the plant on the region. In other words, stability as defined is possible if and only if information can be transported as fast as it is generated by the plant.

In Section IV, the plant is placed in a metric space and the concept of local TFE (LTFE) at a point is defined, by taking appropriate limits in the TFE. Our second main result states that, under certain stabilizability conditions, local uniform asymptotic stabilizability to a fixed point is possible if and only if the data rate exceeds the plant LTFE at that point.

We then focus on deriving a formula for the LTFE of continuously differentiable plants in Euclidean space in Section V. By using real Jordan forms and volume partitioning arguments, we demonstrate that the LTFE of the plant is the sum of the base-2 logarithms of the unstable eigenvalues of the Jacobian at the fixed point. This agrees exactly with results derived previously for noiseless linear plants. We then discuss extensions and open questions in the concluding section.

Throughout this paper, the real numbers are denoted by $\mathbb{R}$, complex numbers $\mathbb{C}$, positive integers $\mathbb{N}$, and nonnegative integers $\mathbb{Z}_{+}$.

\section{TOPOLOGICAL FEEDBACK ENTROPY}

Before discussing the problem of data-rate-limited control, we consider how to quantify the rate at which a dynamical system with inputs generates information. The answer to this question is obviously related to the data-rate problem. However, in this section we define this information rate in an abstract manner that makes no reference to coders, controllers or feedback communication constraints. This underlines its fundamental nature as an intrinsic property of the dynamical system.

As mentioned in the Introduction, Shannon's pioneering work motivated Kolmogorov, and subsequently others, to construct definitions of entropy for completely deterministic nonlinear maps [18]. One of the most general of such constructs is the topological entropy of Adler et al., which applies to continuous maps on compact topological spaces; see [19] and [20] for details. Briefly, the idea behind this definition is to first fix an open cover $\alpha$ for the space, through which each iteration of the map is observed, i.e., all that is known is the sets of the open cover in which the iterations fall. Each observed open set is then inverted to yield an open set in the initial state space. As the number of iterations increases, the family of all possible intersections of initial state open sets forms an increasingly fine open cover for the space. The topological entropy of the map is then obtained by supremizing the asymptotic rate of increase of the cardinality of this open cover over all observation open covers. This in some sense measures the fastest rate at which uncertainty about the initial state can be reduced, or equivalently the fastest rate at which initial state information can be generated.

A later definition of topological entropy by Bowen and Dinaburg [20]-[22]partially extended this to noncompact metric spaces, under the assumption of uniform continuity. However, the purely topological nature of Adler's version is highly attractive, and we will use similar open cover techniques here to construct a definition of TFE. This may appear to be an extension of normal topological entropy to maps on noncompact spaces with inputs, but there is a significant difference in interpretation, as discussed later. Note that the fact that we seek to return the state to a specified compact set via appropriate controls is also the reason that we can use open cover arguments in the style of Adler, even though Bowen's techniques for noncompact metric spaces may appear more directly relevant. We also mention that there is also a body of recent work which explores connections between the entropy of a dynamical system and Bode's sensitivity integral [23]-[25].

We first introduce some basic notation and terminology. Let $X$ be a space endowed with some topology $\mathcal{T}$. A (possibly uncountable) collection $\alpha$ of open sets is called an open cover of a compact set $K \subset X$ if $K \subseteq \cup_{A \in \alpha} A$. By the topological definition of compactness there then exists a finite subcover of $\alpha$, i.e., a finite family $\left\{A_{p}: 1 \leq p \leq r\right\} \subseteq \alpha$ which also covers $K$, and let $N(\alpha \mid K)$ denote the minimum cardinality of such finite subcovers.

Now, consider the fully observed, time-invariant, dynamical system

$$
x_{k+1}=F\left(x_{k}, u_{k}\right) \equiv F_{u_{k}}\left(x_{k}\right) \quad \forall k \in \mathbb{Z}_{+}
$$

where the state $x_{k} \in X$, the input $u_{k} \in$ a set $U$ and $F(\cdot, u) \equiv$ $F_{u}$ is continuous $\forall u \in U$. We analyze this system on a compact set $K$ with nonempty interior, under two alternative conditions of increasing strength.

WI) (Weak Invariability) $K$ can be made weakly invariant under $F$, i.e., there exists $t \in \mathbb{N}$ and a compact $K^{\prime} \subseteq$ int $K$ s.t. $\forall x_{0} \in K \exists$ an input sequence $\left\{H_{k}\left(x_{0}\right)\right\}_{k=0}^{t-\overline{1}}$ in $U$ which ensures $x_{t} \in \operatorname{int} K^{\prime}$.

SI) (Strong Invariability) $K$ can be made strongly invariant under $F$, i.e., there exists a compact $K^{\prime} \subseteq$ int $K$ s.t. $\forall x_{0} \in K \exists$ an input $H_{0}\left(x_{0}\right) \in U$ which ensures $x_{1} \in \operatorname{int} K^{\prime}$.

We remark that for the purposes of this section and the subsequent one, it would be possible to weaken these definitions to only require that the subsequent state $x_{t}$ or $x_{1}$ lies in the interior of $K$, without positing the existence of an inner set $K^{\prime}$. However, the more stringent definition of strong invariability above, in particular, is needed in the proofs of Section IV, and for the sake of a uniform approach an analagous definition has been adopted for weak invariability.

Let $\alpha$ be an open cover of $K, \tau$ a positive integer, and $G=$ $\left\{G_{k}: \alpha \rightarrow U\right\}_{k=0}^{\tau-1}$ a sequence of $\tau$ maps that assign input 
variables to all sets in $\alpha$. Depending on which of the aforementioned invariability conditions holds, impose the following corresponding constraint on $\alpha, \tau$, and $G$.

WI') $\exists$ compact set $K^{\prime} \subseteq \operatorname{int} K$ s.t. $\forall x_{0} \in A \in \alpha$, the sequence of inputs $G(A)$ yields $x_{\tau} \in \operatorname{int} K^{\prime}$, i.e.,

$$
F_{G_{\tau-1}(A)} \ldots F_{G_{0}(A)}(A) \subseteq \operatorname{int} K^{\prime} \quad \forall A \in \alpha .
$$

SI') $\exists$ compact set $K^{\prime} \subseteq \operatorname{int} K$ s.t. $\forall x_{0} \in A \in \alpha$, the sequence of inputs $G(A)$ yields $x_{k} \in \operatorname{int} K^{\prime}, \forall k \in$ $[1, \ldots, \tau]$. That is, $\forall A \in \alpha, k \in[1, \ldots, \tau]$

$$
F_{G_{k-1}(A)} \cdots F_{G_{0}(A)}(A) \subseteq \operatorname{int} K^{\prime} .
$$

To demonstrate the feasibility of WI'), set $\tau$ equal to the invariance time $t$ in assumption WI). By the continuity of $F_{u}$ and the openness of int $K^{\prime}, \forall y \in K$ there is an open set $S(y) \ni y$ s.t. $F_{H_{\tau-1}(y)} \ldots F_{H_{0}(y)}(x) \in \operatorname{int} K^{\prime}, \forall x \in S(y)$. The open cover $\alpha$ is then chosen to be a finite subcover $\left\{L^{1}, \ldots, L^{r}\right\}$ of the open cover $\{S(y) \mid y \in K\}$, guaranteed to exist by the compactness of $K$. Let $y^{q}$ be a point in $K$ such that $L^{q}=S\left(y^{q}\right)$ and set

$$
G_{k}\left(L^{q}\right)=H_{k}\left(y^{q}\right) \quad \forall q \in[1, \ldots, r], k \in[0, \ldots, \tau-1] .
$$

By the preceding observations, we then automatically have that if $x \in L^{q} \in \alpha$, then $F_{G_{\tau-1}\left(L^{q}\right)} \ldots F_{G_{0}\left(L^{q}\right)}(x)=$ $F_{H_{\tau-1}\left(y^{q}\right)} \ldots F_{H_{0}\left(y^{q}\right)}(x) \in \operatorname{int} K^{\prime}$. This confirms the feasibility of constraint WI') under assumption WI). A similar argument demonstrates the feasibility of SI') under SI), where the functions $H_{1}, \ldots, H_{\tau-1}$ are defined recursively in terms of $F$ and $H_{0}$ by

$$
H_{k}\left(x_{0}\right) \triangleq H_{0}\left(x_{k}\right) \quad x_{k}=F_{H_{0}\left(x_{k-1}\right)}\left(x_{k-1}\right)
$$

$\forall x_{0} \in K, k \in[1, \ldots, \tau-1]$, with $\tau$ being any positive integer.

Next, let $A_{0}, A_{1}, A_{2}, \ldots$ be elements of $\alpha$ and define

$$
\begin{aligned}
B_{j} & :=\left\{x_{0} \in X: x_{i \tau} \in A_{i},\left\{u_{l}\right\}_{l=(i-1) \tau}^{i \tau-1}=G\left(A_{i-1}\right)\right. \\
\forall i & \in[0, \ldots, j]\} \quad \forall j \in \mathbb{Z}_{+} .
\end{aligned}
$$

In words, the set $B_{j}$ corresponds to the region in which $x_{0}$ is known to lie, given the observations $x_{i \tau} \in A_{i}, 0 \leq i \leq j$, and inputs $G\left(A_{i}\right) \in U^{\tau}, 0 \leq i \leq j-1$. The openness of $B_{j}$ may be explicitly confirmed by writing it as

$$
\begin{aligned}
B_{j}=A_{0} \cap \Phi_{G\left(A_{0}\right)}^{-1}\left(A_{1}\right) \cap \Phi_{G\left(A_{0}\right)}^{-1} \Phi_{G\left(A_{1}\right)}^{-1}\left(A_{2}\right) \cap \cdots \\
\cdots \cap \Phi_{G\left(A_{0}\right)}^{-1} \cdots \Phi_{G\left(A_{j-1}\right)}^{-1}\left(A_{j}\right) \quad \forall j \in \mathbb{Z}_{+}
\end{aligned}
$$

since the composition $\Phi_{G(A)} \triangleq F_{G_{\tau-1}(A)} \ldots F_{G_{0}(A)}$ is continuous.

Furthermore, every $x_{0} \in K$ must lie in a set $B_{j}$ of the form (5). To see this, observe that if $x_{0} \in K$ then it must lie in some open set $A_{0} \in \alpha$. By the constraint WI') or SI'), the sequence $G\left(A_{0}\right)$ of $\tau$ inputs then forces $x_{\tau} \in K$. Repeating this process indefinitely, it is clear that $\forall x_{0} \in K$ there is a sequence $A_{0}, A_{1}, \ldots$ of sets in $\alpha$ such that $x_{i \tau} \in A_{i}$ with an input sequence $\left\{u_{l}\right\}_{l=(i-1) \tau}^{i \tau-1}=G\left(A_{i-1}\right), \forall i \in[0, \ldots, j-1]$. Hence, $\forall j \in \mathbb{Z}_{+}$

$$
\beta_{j} \triangleq\left\{B_{j}: A_{0}, \ldots, A_{j} \in \alpha\right\}
$$

is an open cover for $K$. As no set $B_{j}$ in a minimal subcover of $\beta_{j}$ is contained in a union of other sets, each carries new information. Hence, as $N\left(\beta_{j} \mid K\right)$ increases, so does the amount of information gained about the initial state. The asymptotic rate of information generation of $F$ on $K$ with inputs in $U$, relative to a given triple $(\alpha, \tau, G)$, may thus be measured by

$$
\lim _{j \rightarrow \infty} \frac{\log _{2} N\left(\beta_{j} \mid K\right)}{j \tau}=\inf _{j \in \mathbb{Z}_{+}} \frac{\log _{2} N\left(\beta_{j} \mid K\right)}{j \tau} .
$$

To verify that the limit on the RHS above exists and equals the infimum, we appeal to a subadditivity theorem of [26]; see also [27]. Observe from (6) that $\forall j, k \in \mathbb{Z}_{+}, \beta_{j+k}$ consists of all sets of the form

$$
\begin{aligned}
B_{j+k}= & {\left[A_{0} \cap \Phi_{G\left(A_{0}\right)}^{-1}\left(A_{1}\right) \cap \cdots \cap \Phi_{G\left(A_{0}\right)}^{-1} \cdots \Phi_{G\left(A_{j-1}\right)}^{-1}\left(A_{j}\right)\right] } \\
& \cap \Phi_{G\left(A_{0}\right)}^{-1} \cdots \Phi_{G\left(A_{j}\right)}^{-1}\left(A_{j+1} \cap \Phi_{G\left(A_{j+1}\right)}^{-1}\left(A_{j+2}\right) \cap \cdots\right. \\
& \left.\cdots \cap \Phi_{G\left(A_{j+1}\right)}^{-1} \cdots \Phi_{G\left(A_{j+k-1}\right)}^{-1}\left(A_{j+k}\right)\right)
\end{aligned}
$$

with $A_{0}, \ldots, A_{j+k}$ running freely over $\alpha$. Note that the term inside the square brackets runs over all sets in the open cover $\beta_{j}$, while the argument inside the large parentheses runs over all elements of $\beta_{k-1}$. Now, constrain $\left\{A_{i}\right\}_{i=0}^{j}$ to index sets in a minimal subcover $\beta_{j}^{\prime}$ of $\beta_{j}$, and $\left\{A_{i}\right\}_{i=j+1}^{j+k}$ to index those in a minimal subcover $\beta_{k-1}^{\prime}$ of $\beta_{k-1}$. Denote the constrained family of sets $B_{j+k}$ thus formed by $\beta_{j+k}^{*}$. To see that $\beta_{j+k}^{*}$ is still an open cover for $K$, observe that any $x \in K$ must lie in a set $B_{j}^{\prime} \in \beta_{j}^{\prime}$ indexed by some sequence $\left\{A_{i}\right\}_{i=0}^{j}$ in $\alpha$. Furthermore, $\Phi_{G\left(A_{j}\right)} \ldots \Phi_{G\left(A_{0}\right)}(x) \in K$ and, thus, lies in some set $B_{k-1}^{\prime}$ in the minimal subcover $\beta_{k-1}^{\prime}$. Hence, $x \in \Phi_{G\left(A_{0}\right)}^{-1} \cdots \Phi_{G\left(A_{j}\right)}^{-1}\left(B_{k-1}^{\prime}\right)$, and so $\beta_{j+k}^{*}$ is still a cover for $K$. As there are $N\left(\beta_{j} \mid K\right)$ sets in $\beta_{j}^{\prime}$, and to each there correspond $N\left(\beta_{k-1} \mid K\right)$ possible sets $\Phi_{G\left(A_{0}\right)}^{-1} \ldots \Phi_{G\left(A_{j}\right)}^{-1}\left(B_{k-1}^{\prime}\right)$, the number of distinct elements in $\beta_{j+k}^{*}$ cannot exceed $N\left(\beta_{j} \mid K\right) N\left(\beta_{k-1} \mid K\right)$. By the definition of minimal subcovers and the monotonicity of $N\left(\beta_{k} \mid K\right)$, we must then have that $\forall j, k \in \mathbb{Z}_{+}$

$N\left(\beta_{j+k} \mid K\right) \leq N\left(\beta_{j} \mid K\right) N\left(\beta_{k-1} \mid K\right) \leq N\left(\beta_{j} \mid K\right) N\left(\beta_{k} \mid K\right)$.

Polya and Szego's subadditivity theorem [27] then states that the limit in (8) exists and is precisely equal to the infimum.

Infimizing (8) again over $(\alpha, \tau, G)$, the weak invariance TFE of $F$ on $K$ with inputs in $U$ is finally defined as

$$
\begin{aligned}
h^{\mathrm{wi}}(F, K, U) & :=\inf _{\alpha, \tau, G} \lim _{j \rightarrow \infty} \frac{\log _{2} N\left(\beta_{j} \mid K\right)}{j \tau}, \\
& =\inf _{\alpha, \tau, G, j} \frac{\log _{2} N\left(\beta_{j} \mid K\right)}{j \tau}
\end{aligned}
$$

under constraint WI') on $\alpha, \tau, G$, and assumption WI) on $F, K$, $U$. Analogously, the strong invariance TFE is given by

$$
\begin{aligned}
h^{\mathrm{si}}(F, K, U) & :=\inf _{\alpha, G, \tau} \lim _{j \rightarrow \infty} \frac{\log _{2} N\left(\beta_{j} \mid K\right)}{j \tau}, \\
& =\inf _{\alpha, \tau, G, j} \frac{\log _{2} N\left(\beta_{j} \mid K\right)}{j \tau}
\end{aligned}
$$

under constraint $\mathrm{SI}^{\prime}$ ) on $\alpha, G$ and assumption SI) on $F, K$, and $U$. As the constraint SI') is more restrictive than WI'), it follows that $h^{\mathrm{si}}(F, K, U) \geq h^{\mathrm{wi}}(F, K, U)$. 
The infimum in the definitions of TFE suggests that it can be interpreted as the smallest rate at which initial state information can be generated while maintaining invariance. In contrast, the standard definition(s) of topological entropy measure the largest rate at which a fixed map can generate initial state information. This distinction is apparent for the simple example of the tent map on the unit interval with an additive control term

$$
F(x, u)=2 \min \{x, 1-x\}+u \quad \forall x \in K=[0,1] .
$$

As $F(x, 0) \in \operatorname{int} K$ for any $x \in \operatorname{int} K$, it is easy to show that the TFE for weak and strong invariance are both 0 . On the other hand, the topological entropy of this map with $u=0$ is strictly positive (in fact $1 \mathrm{bit} / \mathrm{sample}$ ).

Observe that we trivially have the monotonicity property

$$
h^{\mathrm{wi}(\mathrm{si})}(F, K, U) \geq h^{\mathrm{wi}(\mathrm{si})}\left(F, K, U^{\prime}\right) \quad \forall U^{\prime} \supset U
$$

since the inputs can have more possible values and thereby reduce the infimum in the definitions of TFE. However, a similar monotonicity property cannot be deduced for a compact $J \subset K$ satisfying condition [WI] ([SI]) with the same input set $U$. Even though an open cover $\alpha$ for $K$ is automatically an open cover for $J$, it may not satisfy the constraint WI') [SI')] on the infimization with the same $G$ and $\tau$. Thus we can not conclude that TFE increases as the state region shrinks.

The feedback entropies introduced above are defined purely in terms of open sets for a very general class of maps with inputs. As we have made no reference whatsoever to coding and control structures, feedback communication constraints, or other external constructs, topological feedback entropy as defined above is an inherent property of the dynamical system (1) alone. In the next section, we close the feedback loop and discuss the significance of TFE in the particular context of data-rate-limited control. This leads to an alternative and perhaps more practically relevant characterization of TFE.

\section{INVARIANCE UNDER DATA-RATE-LIMITED FEEDBACK}

In this section, we investigate a class of nonlinear control problems that involve limited data rates in the feedback loop. As discussed in the introduction, such a communication constraint has a negative effect on the attainable control performance. Different approaches to analyzing and mitigating this effect have recently been described in [28] and [29]. Our specific interest here is characterizing the smallest possible data rate that permits a specified compact set to be made invariant, by a causal coding and control law belonging to a general class. We then show that, in this context, the infimum data rate is precisely equal to the intrinsic information rate of the plant, as measured by its TFE.

Let the plant be given by (1). Suppose that a sensor measures its states and is connected to a controller by a noiseless digital channel which carries one discrete-valued symbol $s_{k}$ per sampling interval, selected from a coding alphabet $S_{k}$ of timevarying size $\mu_{k}$. The transmission data rate $R$ of the channel may then be defined as the asymptotic average bit rate

$$
R \triangleq \liminf _{k \rightarrow \infty} \frac{1}{k} \sum_{j=0}^{k-1} \log _{2} \mu_{j} .
$$

For technical reasons, we impose the mild requirement that $\log _{2} \mu_{k} / k \rightarrow 0$ as $k \rightarrow \infty$. Suppose that each symbol transmitted by the coder may depend on all past and present states and past symbols, i.e.,

$$
s_{k}=\gamma_{k}\left(\left\{x_{i}\right\}_{i=0}^{k},\left\{s_{i}\right\}_{i=0}^{k-1}\right) \in S_{k} \quad \forall k \in \mathbb{Z}_{+}
$$

where $\gamma_{k}: X^{k+1} \times S_{0} \times \cdots \times S_{k-1} \rightarrow S_{k}$ is the coder mapping at time $k$. In practice, a finite-dimensional coder would obviously be desired, but the more general formulation shown previously allows us to focus on the limitations imposed by the digital link. Assuming that the digital channel is errorless, at time $k$ the controller has $s_{0}, \ldots, s_{k}$ available and generates

$$
u_{k}=\delta_{k}\left(\left\{s_{i}\right\}_{i=0}^{k}\right) \in U \quad \forall k \in \mathbb{Z}_{+}
$$

where $\delta_{k}: S_{0} \times \cdots \times S_{k} \rightarrow U$ is the controller function at time $k$.

Define the coder-controller as the triple of alphabet, coder and controller sequences $(S, \gamma, \delta) \triangleq \quad\left(\left\{S_{k}\right\}_{k \in \mathbb{Z}_{+}},\left\{\gamma_{k}\right\}_{k \in \mathbb{Z}_{+}},\left\{\delta_{k}\right\}_{k \in \mathbb{Z}_{+}}\right)$. If for some $r \in \mathbb{N}$ and all $k \in \mathbb{Z}_{+}$the coder-controller satisfies

$$
\begin{aligned}
S_{k}= & S_{k \bmod r} \\
\gamma_{k}\left(\left\{x_{i}\right\}_{i=0}^{k},\left\{s_{i}\right\}_{i=0}^{k-1}\right)= & \gamma_{k \bmod r}\left(\left\{x_{i}\right\}_{i=r\lfloor k / r\rfloor}^{k},\right. \\
& \left.\left\{s_{i}\right\}_{i=r\lfloor k / r\rfloor}^{k-1}\right) \\
\delta_{k}\left(\left\{s_{i}\right\}_{i=0}^{k}\right)= & \delta_{k \bmod r}\left(\left\{s_{i}\right\}_{i=r\lfloor k / r\rfloor}^{k}\right)
\end{aligned}
$$

we call it periodic with period $r$. Coding and control equations of this class are easier to implement, due to the finite memory requirement, and are also useful analytically elsewhere in this paper.

Given an asymptotic average data rate $R \geq 0$, our objective is to investigate whether there exists a coder-controller, with inputs in $U$, which renders $K$ either

1) weakly invariant, i.e., $\exists \tau \in \mathbb{N}$ and compact $K^{\prime} \subseteq \operatorname{int} K$ s.t. $x_{j \tau} \in \operatorname{int} K^{\prime}, \forall x_{0} \in K, j \in \mathbb{N}$; or

2) strongly invariant, i.e., $\exists$ compact $K^{\prime} \subseteq \operatorname{int} K$ s.t. $x_{k} \in$ $\operatorname{int} K^{\prime}, \forall x_{0} \in K, k \in \mathbb{N}$.

We now state the first major result of this paper.

Theorem 1: Consider the continuous-w.r.t.-state plant $F$ (1), with states in a topological space $X$, inputs lying in a set $U$ and initial state in some compact region $K$ with nonempty interior. Assume that the weak (strong) invariability assumption WI) [respectively, SI)] holds on $F, K, U$.

For $K$ to be made weakly (strongly) invariant by a coder-controller of the form (13)-(14), the feedback data rate $R$ (12) cannot be less than the weak (strong) invariance topological feedback entropy (9) [(10)] of the plant on $K$ with input set $U$

$$
R \geq h^{\mathrm{wi}(\mathrm{si})}(F, K, U)
$$

Furthermore, this lower bound is tight, i.e., there exist codercontrollers that achieve weak (strong) invariance at data rates arbitrarily close to it.

This theorem relates the practical problem of data-rate-limited feedback to the abstract concept of topological feedback 
entropy. Recalling that the TFE of a plant measures the rate at which it generates feedback information, the result above states that invariance is possible if and only if information can be transported across the channel as fast as it is generated. This is similar to the role that Shannon's source coding theorem plays in information theory and digital communications [16].

In light of the previous theorem, it is tempting to simply define feedback entropy as the smallest data rate which permits invariance. However, the problem with such an operational definition is that it is tied to the specific assumptions imposed on the the digital channel, the coder, and the controller. In contrast, TFE is completely independent of all external constructs and is, thus, a purer measure of the intrinsic information rate of the plant. Again, there is an analogy with source coding, where the entropy of a statistical source is defined axiomatically in terms of a functional on its distribution, instead of the smallest data rate for "reliable" communications. As mentioned in the introduction, this avoids the problems associated with different formulations of coding and "reliability".

In the remainder of this section, we present a proof of this theorem. For conciseness only weak invariance is considered; the proof for strong invariance is nearly identical. To reduce clutter, the wi superscripts and $(F, K, U)$ arguments are dropped.

\section{A. Necessity of Lower Bound}

We prove the lower bound (16) by basically showing that any coder-controller which achieves weak invariance with rate $R$ induces an open cover $\alpha$, mapping sequence $G$, and recurrence time $\tau$, as defined in the previous section, with entropy rate also $\approx R$.

For any such coder-controller $(S, \gamma, \delta)$, there exist $\tau_{0} \in \mathbb{N}$ and compact $K^{\prime} \subset \operatorname{int} K$ s.t. $\forall x_{0} \in K$ and $j \in \mathbb{N}, x_{j \tau_{0}} \in$ $\operatorname{int} K^{\prime}$. It then follows from (12) that $\forall \varepsilon>0$, there exist infinitely many $k^{\prime} \in \mathbb{N}$ s.t.

$$
\frac{1}{k^{\prime}} \sum_{k=0}^{k^{\prime}-1} \log _{2} \mu_{k}<R+\frac{\varepsilon}{2} .
$$

Set $l=\left\lceil k^{\prime} / \tau_{0}\right\rceil$, so that $k^{\prime}=l \tau_{0}-q$ for some $q \in\left[0, \ldots, \tau_{0}-1\right]$. Then

$$
\begin{aligned}
\frac{1}{l \tau_{0}} \sum_{k=0}^{l \tau_{0}-1} \log _{2} \mu_{k} & =\frac{1}{k^{\prime}+q} \sum_{k=0}^{k^{\prime}-1+q} \log _{2} \mu_{k} \\
& =\frac{1}{k^{\prime}+q} \sum_{k=0}^{k^{\prime}-1} \log _{2} \mu_{k}+\frac{1}{k^{\prime}+q} \sum_{k=k^{\prime}}^{k^{\prime}-1+q} \log _{2} \mu_{k} \\
& \leq \frac{1}{k^{\prime}} \sum_{k=0}^{k^{\prime}-1} \log _{2} \mu_{k}+\frac{1}{k^{\prime}+q} \sum_{k=k^{\prime}}^{k^{\prime}-1+q} \log _{2} \mu_{k} \\
& <R+\frac{\varepsilon}{2}+\frac{\tau_{0}-1}{k^{\prime}+\tau_{0}-1} \max _{i \in\left[0, . ., \tau_{0}-1\right]} \log _{2} \mu_{k^{\prime}+i} \\
& <R+\varepsilon
\end{aligned}
$$

for $k^{\prime}$ sufficiently large, since $\log _{2} \mu_{k} / k \rightarrow 0$. Let $r=l \tau_{0}$. Evidently, $x_{r} \in \operatorname{int} K^{\prime} \subset K$, so if we "reset the clock" to zero and perform the coding and control again with initial state $=$ $x_{r}$ we then get a new state $\in \operatorname{int} K^{\prime}$ at time $2 r$.
This resetting process can be repeated, so by induction we can thus convert the original, possibly recursive, coder-controller $(S, \gamma, \delta)$ into a periodic coder-controller $\left(S^{\mathrm{p}}, \gamma^{\mathrm{p}}, \delta^{\mathrm{p}}\right)$ with pe$\operatorname{riod} r$. The most important point about this conversion is that the new coder-controller achieves $\tau_{0}$-invariance with average data rate within $\varepsilon$ of the original (NB: period $\neq$ invariance time). The alphabet and coding and control equations of the periodic scheme are formally given $\forall k \in \mathbb{Z}_{+}$by

$$
\begin{aligned}
S_{k}^{\mathrm{p}} & =S_{k \bmod r} \\
s_{k} & =\gamma_{k}^{\mathrm{p}}\left(\left\{x_{i}\right\}_{i=0}^{k},\left\{s_{i}\right\}_{i=0}^{k-1}\right) \\
& =\gamma_{k \bmod r}\left(\left\{x_{i}\right\}_{i=r\lfloor k / r\rfloor}^{k},\left\{s_{i}\right\}_{i=r\lfloor k / r\rfloor}^{k-1}\right) \\
u_{k} & =\delta_{k}^{\mathrm{p}}\left(\left\{s_{i}\right\}_{i=0}^{k}\right)=\delta_{k \bmod r}\left(\left\{s_{i}\right\}_{i=r\lfloor k / r\rfloor}^{k}\right) .
\end{aligned}
$$

Observe that with the coder-controller fixed as above, the symbol sequence $\left\{s_{k}\right\}_{k=j r}^{(j+1) r-1}$ is completely determined by $x_{j r}, \forall j \in \mathbb{Z}_{+}$, by a fixed map that incorporates both coder and controller functions. Thus, $\forall j \in \mathbb{Z}_{+}$

$$
\begin{aligned}
\left\{s_{k}\right\}_{k=j r}^{(j+1) r-1} & \equiv \Gamma\left(x_{j r}\right) \\
\left\{u_{k}\right\}_{k=j r}^{(j+1) r-1} & \equiv \Delta\left(\left\{s_{k}\right\}_{k=j r}^{(j+1) r-1}\right)
\end{aligned}
$$

where $\Gamma, \Delta$ are mappings that do not change with $j$.

Now, consider the disjoint regions $\Gamma^{-1}\left(\left\{c_{k}\right\}_{k=0}^{r-1}\right) \subseteq K$ as the symbol sequence $\left\{c_{k}\right\}_{k=0}^{r-1}$ varies over all possible sequences in $S_{0} \times \cdots \times S_{r-1}$. The total number of distinct symbol sequences is just $\prod_{k=0}^{r-1} \mu_{k}$, so the total number $n$ of nonempty and distinct regions must be less than or equal to this. Denote these coding regions by $C^{1}, \ldots, C^{n}$, noting that $K \subseteq \cup_{i=1}^{n} C^{i}$. We can then rewrite the control equation in (18) as

$$
\left\{u_{k}\right\}_{k=j r}^{(j+1) r-1}=\Delta^{*}\left(C^{i}\right) \text { if } x_{j r} \in C^{i}
$$

by defining the map $\Delta^{*}\left(C^{i}\right)=\Delta\left(\left\{c_{k}\right\}_{k=0}^{r-1}\right)$ iff $C^{i}=$ $\Gamma^{-1}\left(\left\{c_{k}\right\}_{k=0}^{r-1}\right)$.

We are now in a position to construct the $\tau \in \mathbb{N}$, the open cover $\alpha$ and the mapping sequence $G=\left\{G_{k}: \alpha \rightarrow U\right\}_{k=0}^{\tau-1}$ required in the definition of TFE. Set $\tau=r$ and then construct the open cover $\alpha$ as follows. Observe that $\forall x_{0} \in$ any coding region $C^{i}$

$$
\Phi_{\Delta^{*}\left(C^{i}\right)}\left(x_{0}\right) \in \operatorname{int} K^{\prime} \quad \forall x_{0} \in C^{i}
$$

where the left-hand side denotes the dynamical map $F_{u}$ applied $\tau=r$ times with the input sequence (19). By the continuity of $F_{u}$ (hence of $\Phi_{\Delta^{*}\left(C^{i}\right)}$ ) and the openness of int $K^{\prime}$, it then follows that $\forall x_{0} \in C^{i}$, there is an open set $S\left(x_{0}\right) \ni x_{0}$ s.t. $\Phi_{\Delta^{*}\left(C^{i}\right)}(y) \in \operatorname{int} K^{\prime}, \forall y \in S\left(x_{0}\right)$. In this way we can construct $\forall i \in[1, \ldots, n]$ an open set $L^{i}=\cup_{x_{0} \in C^{i}} S\left(x_{0}\right)$ s.t.

$$
\Phi_{\Delta^{*}\left(C^{i}\right)}(y) \in \operatorname{int} K^{\prime}, \forall y \in L^{i} \Leftrightarrow \Phi_{\Delta^{*}\left(C^{i}\right)}\left(L^{i}\right) \subseteq \operatorname{int} K^{\prime} .
$$

As $C^{i} \subseteq L^{i}$ and $K \subseteq \cup_{i=1}^{n} C^{i}, \alpha=\left\{L^{i}: i=1, \ldots, n\right\}$ is an open cover for $K$. Finally, construct the mapping sequence $G$ as

$$
G_{k}\left(L^{i}\right)=\Delta^{*}\left(C^{i}\right), \forall L^{i} \in \alpha, \quad k=0, \ldots, \tau-1 .
$$

Substituting this construction into (20), it is then evident that the constraint (WI') on $\tau, \alpha$, and $G$ is satisfied. 
We now generate the (finite) open covers $\beta_{j}$ of (7). Observe that the minimum cardinality $N\left(\beta_{j} \mid K\right)$ of a subcover of $\beta_{j} \leq$ the number of sets in $\beta_{j}$, which by (7) must be $\leq$ the number of possible sequences $\left\{A_{i}\right\}_{i=0}^{j}$ of sets in $\alpha$, i.e.,

$$
\begin{aligned}
N\left(\beta_{j} \mid K\right) & \leq n^{j+1} \Rightarrow \lim _{j \rightarrow \infty} \frac{\log _{2} N\left(\beta_{j} \mid K\right)}{j} \leq \log _{2} n \\
\Rightarrow h & \equiv \inf _{\tau, \alpha, G} \lim _{j \rightarrow \infty} \frac{\log _{2} N\left(\beta_{j} \mid K\right)}{j \tau} \leq \frac{\log _{2} n}{\tau} \\
& \leq \frac{\log _{2}\left(\prod_{k=0}^{\tau-1} \mu_{k}\right)}{\tau}=\frac{1}{\tau} \sum_{k=0}^{\tau-1} \log _{2} \mu_{k} .
\end{aligned}
$$

From (17), we can ensure that $\forall \varepsilon>0$, the last term on the RHS $<R+\varepsilon$ by choosing $\tau \equiv l \tau_{0}$ sufficiently large. Hence, $R \geq h$.

\section{B. Achievability of the Lower Bound}

To prove that data rates arbitrarily close to $h$ can be achieved, we first show that any triple $(\tau, \alpha, G)$ which satisfies constraint WI') in the previous section induces a periodic coder-controller which renders $K \tau$-invariant. The (uninfimized) entropy with respect to such a triple is defined as

$$
H \triangleq \lim _{j \rightarrow \infty} \frac{\log _{2} N\left(\beta_{j} \mid K\right)}{j \tau}
$$

where $\beta_{j}$ is defined by (6) and (7). It then follows that $\forall \varepsilon>$ $0, \exists j \in \mathbb{N}$ s.t.

$$
\frac{\log _{2} N\left(\beta_{j} \mid K\right)}{j \tau} \leq H+\frac{\varepsilon}{2} .
$$

Recalling that $\beta_{j}$ is an open cover for the compact set $K$, select a minimal subcover of $\beta_{j}$ and denote it by $\left\{D^{1}, \ldots, D^{m}\right\}$, where $m=N\left(\beta_{j} \mid K\right)$ by definition. We construct a periodic coding law using these overlapping sets via the rule

$$
s_{k}=\left\{\begin{array}{ll}
\min \left\{i: x_{k} \in D^{i}\right\}, & \text { when } k \in[0, j \tau, 2 j \tau, \ldots) \\
1, & \text { otherwise }
\end{array} .\right.
$$

Evidently, the coding alphabet size $\mu_{k}=m$ when $k$ is a multiple of $j \tau$ and $=1$ otherwise, so the (average) data rate of this coder is simply

$$
R=\frac{\log _{2} m}{j \tau}=\frac{\log _{2} N\left(\beta_{j} \mid K\right)}{j \tau} \leq H+\frac{\varepsilon}{2} .
$$

The next step is to construct the controller from the mapping sequence $G$. By definition, for each set $B_{j} \in \beta_{j}$ there are sets $A_{0}, \ldots, A_{j} \in \alpha$ s.t. (5) holds. Upon receiving the symbol $s_{l j \tau}=i$ which indexes an open set $D^{i}$ containing $x_{l j \tau} \in K^{\prime}$ in the minimal subcover of $\beta_{j}$, the controller finds $\left\{A_{i}\right\}_{i=0}^{j}$ in $\alpha$ which yields $B_{j}=D^{i}$ and then applies control inputs via the periodic rule

$$
\left\{u_{k}\right\}_{k=(l j+q) \tau}^{(l j+q+1) \tau-1}=G\left(A_{q}\right) \quad \forall l \in \mathbb{Z}_{+}, q \in[0, \ldots, j-1] .
$$

By the constraint (2), $x_{(l j+q) \tau} \in \operatorname{int} K^{\prime}$, so that $\tau$-invariance is achieved at rate $R \leq H+\varepsilon / 2$. As $h$ is the infimum of $H, \forall \varepsilon>0$ we can find $(\tau, \alpha, G)$ s.t. $H<h+\varepsilon / 2$. Hence, $R<h+\varepsilon$ and the result follows by choosing $\varepsilon$ arbitrarily small.

The key point about the construction e above is that the coder and controller do not simply index all elements of $\alpha$ and indicate which element of $\alpha$ each state lies in. This demands a data rate equal to $\tau^{-1} \log _{2} N(\alpha \mid K)$ and ignores the fact that not all sequences $\left(A_{0}, \ldots, A_{j}\right)$ occur. A more efficient approach is to generate the initial state cover $\beta_{j}$ and transmit the index of an element of $\beta_{j}$ which contains $x_{l j \tau}, l \in \mathbb{Z}_{+}$. For each $l$-th "cycle" of duration $j \tau$, this suffices to determine sets $A_{q}$ containing $x_{(l j+q) \tau}, q=0, \ldots, j-1$, which can then be used to generate control inputs via the mapping $G$.

Observe as well that the periodic coder-controller constructed above actually achieves a slightly stronger objective than weak invariance, since every point in the open set $L \triangleq \cup D^{i} \supseteq K$ is mapped in $\tau$ instants into the interior of $K^{\prime}$. The strong invariance version of this property will be particularly useful when we discuss asymptotic stability in the next section. For convenience, we state it here without further proof.

Lemma 1: Consider the continuous-w.r.t.-state plant (1) with states in a topological space $X$, and let the compact region $K \subseteq X$ with nonempty interior satisfy the strong invariability assumption SI) with input set $U$.

Then for any $\varepsilon>0$, there exists a periodic coder-controller with data rate

$$
R \leq h^{\mathrm{si}}(F, K, U)+\varepsilon
$$

for which $\exists$ compact $K^{\prime} \subseteq \operatorname{int} K$ and open $L \supseteq K$ such that $x_{k} \in \operatorname{int} K^{\prime}, \forall x_{0} \in L, k \in \mathbb{N}$.

Explicit formulas for the TFE of various plants are somewhat difficult to derive. However, for a controllable linear time-invariant plant $(\mathbf{A}, \mathbf{B})$, theorem 1 and techniques adapted from [11], [12] can be used to show that the TFE for both weak and strong invariability on a set $K$ with unconstrained inputs is just $\sum_{\eta \in \sigma(\mathbf{A}),|\eta| \geq 1} \log _{2}|\eta|$, as expected.

\section{LOCAL TOPOLOGICAL FEEDBACK ENTROPY AND ASYMPTOTIC STABILIZABILITY}

In many control systems, the objective is not just to keep the state of the plant (1) within some compact region $K$ but to steer it asymptotically to a specified point $x_{*}$, using feedback inputs approaching a constant value $u_{*}$. In this section, we construct a local topological feedback entropy (LTFE) to characterize such situations. We then show that the smallest data rate for local uniform asymptotic stabilizability (LUAS) is precisely equal to the LTFE of the plant at the target point.

It is still assumed that $F(\cdot, u)$ is continuous $\forall u \in V$. As local uniform asymptotic stability is a stronger objective than making a compact initial state set invariant, additional assumptions will also be placed on the plant (1), as listed as follows.

A) The state-space $X$ has metric $d_{X}$ and the inputs $u_{k}$ belong to a space $V$ with metric $d_{V}$. 
B) There is a constant input $u_{*} \in V$ and point $x_{*} \in X$ s.t. $x_{*}=F\left(x_{*}, u_{*}\right)$.

C) (Local Strong Invariability) For any $\varepsilon>0, \exists \varrho>0$ s.t. $\forall \varepsilon^{\prime} \in(0, \varrho]$ the state region $K=B_{X}\left(\varepsilon^{\prime}\right) \triangleq\{x \in$ $\left.X: d_{X}\left(x, x_{*}\right) \leq \varepsilon^{\prime}\right\}$ satisfies the strong invariability condition [SI] with an input set $U=B_{V}(\varepsilon) \triangleq\{u \in$ $\left.V: d_{V}\left(u, u_{*}\right) \leq \varepsilon\right\}$.

With these assumptions in place, define the LTFE of the plant at the target point $\left(x_{*}, u_{*}\right)$ as

$$
h_{*}\left(F, x_{*}, u_{*}\right) \triangleq \lim _{\varepsilon \rightarrow 0} \limsup _{\varepsilon^{\prime} \rightarrow 0} h^{\mathrm{si}}\left(F, B_{X}\left(\varepsilon^{\prime}\right), B_{V}(\varepsilon)\right)
$$

under the topology of $X$ induced by its metric, where $h^{\text {si }}$ is the strong invariance TFE defined in (10). Note that since TFE increases with decreasing input sets (11), the outer limit is just a supremum over $\varepsilon>0$ and hence exists in the extended half-line $[0, \infty]$.

We call the plant locally uniformly asymptotically stabilizable (LUAS) at the target point if $\forall \varepsilon>0, \exists \varrho>0$ such that $\forall \varepsilon^{\prime} \in$ $(0, \varrho]$ there is a coder-controller $(S, \gamma, \delta)$ yielding

$$
\begin{aligned}
& \sup _{k \in \mathbb{N}, x_{0} \in B_{X}\left(\varepsilon^{\prime}\right)} d_{X}\left(x_{k}, x_{*}\right)<\varepsilon^{\prime} \\
& \sup _{k \in \mathbb{Z}_{+}, x_{0} \in B_{X}\left(\varepsilon^{\prime}\right)} d_{V}\left(u_{k}, u_{*}\right) \leq \varepsilon \\
& \lim _{k \rightarrow \infty} \sup _{x_{0} \in B_{X}\left(\varepsilon^{\prime}\right)} d_{X}\left(x_{k}, x_{*}\right)=0 \\
& \lim _{k \rightarrow \infty} \sup _{x_{0} \in B_{X}\left(\varepsilon^{\prime}\right)} d_{V}\left(u_{k}, u_{*}\right)=0 .
\end{aligned}
$$

We now state the second main result of this paper.

Theorem 2: Consider the continuous-w.r.t.-state plant (1) under assumptions A)-C) above. Any coder-controller (13) and (14) which locally uniformly asymptotically stabilizes it in the sense (26) must have a data rate $R$ (12) not less than the local topological feedback entropy (25) of the plant at the target point $\left(x_{*}, u_{*}\right)$

$$
R \geq h_{*}\left(F, x_{*}, u_{*}\right) .
$$

Furthermore, this lower bound is tight, i.e., there exist codercontrollers at data rates arbitrarily close to it that achieve (26). $\nabla$

Like Theorem 1, this result states that local asymptotic stabilizability is possible if and only if the rate at which information can circulate in the feedback loop is greater than the rate at which the plant generates initial state information at the target point. We now present the proof of necessity and then sufficiency.

\section{A. Necessity}

Suppose that the plant is locally stabilizable in the sense (26) at data rate $R$. For any arbitrary $\varepsilon>0$, we can then find $\varrho>$ 0 such that for any $\varepsilon^{\prime} \leq \varrho$ there is an $\varepsilon_{0}<\varepsilon^{\prime}$ and a codercontroller s.t. $\forall k \in \mathbb{Z}_{+}, x_{0} \in B_{X}(\varepsilon)$

$$
x_{k+1} \in B_{X}\left(\varepsilon_{0}\right) \subset \operatorname{int} B_{X}\left(\varepsilon^{\prime}\right), \quad u_{k} \in B_{V}(\varepsilon) .
$$

In other words, the coder-controller makes $K=B_{X}\left(\varepsilon^{\prime}\right)$ strongly invariant with controls in $B_{V}(\varepsilon)$. By Theorem 1, the data rate cannot be less then the strong invariance TFE of the plant with $K=B_{X}\left(\varepsilon^{\prime}\right)$ and $U=B_{V}(\varepsilon)$

$$
R \geq h^{\mathrm{si}}\left(F, B_{X}\left(\varepsilon^{\prime}\right), B_{V}(\varepsilon)\right) .
$$

By definition of LTFE (25), $\forall \vartheta>0$, we can find arbitrarily small $\varepsilon$ such that

$$
\limsup _{\varepsilon^{\prime} \rightarrow 0} h^{\mathrm{si}}\left(F, B_{X}\left(\varepsilon^{\prime}\right), B_{V}(\varepsilon)\right) \geq h_{*}\left(F, x_{*}, u_{*}\right)-\vartheta
$$

so there must exist arbitrarily small $\varepsilon^{\prime}$ such that

$$
h^{\mathrm{si}}\left(F, B_{X}\left(\varepsilon^{\prime}\right), B_{V}(\varepsilon)\right) \geq h_{*}\left(F, x_{*}, u_{*}\right)-\vartheta .
$$

Substituting this into (28)

$$
R \geq h_{*}\left(F, x_{*}, u_{*}\right)-\vartheta .
$$

As $\vartheta$ can be made arbitrarily small, it follows that $R \geq$ $h_{*}\left(F, x_{*}, u_{*}\right)$.

\section{B. Sufficiency}

We now prove that the data rate lower bound (27) is tight. By definition (25) of LTFE and the monotonicity of $h^{\text {si }}$ with respect to input sets (11)

$$
\limsup _{\varepsilon^{\prime} \rightarrow 0} h^{\mathrm{si}}\left(F, B_{X}\left(\varepsilon^{\prime}\right), B_{V}(\varepsilon)\right) \leq h_{*}\left(F, x_{*}, u_{*}\right) \quad \forall \varepsilon>0 .
$$

As such, $\forall \theta, \varepsilon>0 \exists \varrho_{0}>0$ such that $\forall \varepsilon^{\prime} \in\left(0, \varrho_{0}\right]$

$$
h^{\mathrm{si}}\left(F, B_{X}\left(\varepsilon^{\prime}\right), B_{V}(\varepsilon)\right) \leq h_{*}\left(F, x_{*}, u_{*}\right)+\frac{\theta}{2} .
$$

Setting $\varrho_{1}=$ the minimum of $\varrho_{0}$ and the $\varrho$ of the local strong invariability condition $C$ ), Lemma 1 then states that for each $\varepsilon^{\prime} \in\left(0, \varrho_{1}\right]$ there exists an open set $L \supset B_{X}\left(\varepsilon^{\prime}\right)$, a compact set $K^{\prime} \subset \operatorname{int} B_{X}\left(\varepsilon^{\prime}\right)$, and a periodic coder-controller such that any initial state in $L$ is driven into $K^{\prime}$ at every subsequent instant. Furthermore, this coder-controller can be chosen to have data rate

$$
R \leq h^{\mathrm{si}}\left(F, B_{X}\left(\varepsilon^{\prime}\right), B_{V}(\varepsilon)\right)+\frac{\theta}{2}<h_{*}\left(F, x_{*}, u_{*}\right)+\theta .
$$

Now, $\forall \varepsilon, \theta>0, \varepsilon^{\prime} \in\left(0, \varrho_{1}\right]$, define the set $\mathcal{F}\left(\varepsilon, \varepsilon^{\prime}, \theta\right)$ of finitely switched periodic coder-controllers as follows. For each $(S, \gamma, \delta) \in \mathcal{F}\left(\varepsilon, \varepsilon^{\prime}, \theta\right)$ there is a finite, strictly increasing sequence of nonnegative switching times $\left\{M_{l}\right\}_{l=0}^{N-1}$ and corresponding periods $\left\{T_{l}\right\}_{l=0}^{N-1}$, with $M_{l+1}=M_{l} \bmod T_{i}$, such that at any time $k \in\left[M_{l}, \ldots, M_{l+1}-1\right]$, the coder-controller satisfies

$$
\begin{aligned}
S_{k}= & S_{M_{l}+\left[\left(k-M_{l}\right) \bmod T_{l}\right]} \\
s_{k}= & \gamma_{M_{l}+\left[\left(k-M_{l}\right) \bmod T_{l}\right]}\left(\left\{x_{i}\right\}_{i=M_{l}+T_{l}\left\lfloor\left(k-M_{l}\right) / T_{l}\right\rfloor}^{k}\right. \\
& \left.\left\{s_{i}\right\}_{\left.i=M_{l}+T_{l}\left\lfloor\left(k-M_{l}\right) / T_{l}\right\rfloor\right)}^{k-1}\right) \\
u_{k}= & \delta_{M_{l}+\left[\left(k-M_{l}\right) \bmod T_{l}\right]}\left(\left\{s_{i}\right\}_{\left.i=M_{l}+T_{l}\left\lfloor\left(k-M_{l}\right) / T_{l}\right\rfloor\right) .}^{k}\right.
\end{aligned}
$$


In other words, the coder-controller is periodic with period $T_{l}$ on each interval $\left[M_{l}, \ldots, M_{l+1}-1\right]$, where for convenience $M_{0}=$ $0, M_{N}=\infty$. Furthermore, let each $\left.(S, \gamma, \delta) \in \mathcal{F}\left(\varepsilon, \varepsilon^{\prime}, \theta\right) i\right)$ have average data rate satisfying (30), and ii) render the initial state ball $B_{X}\left(\varepsilon^{\prime}\right)$ strongly invariant using control inputs $u_{k} \in$ $B_{V}(\varepsilon)$.

Now, let

$$
\zeta \triangleq \inf _{(S, \gamma, \delta) \in \mathcal{F}\left(\varepsilon, \varepsilon^{\prime}, \theta\right)} \limsup _{k \rightarrow \infty} \sup _{x_{0} \in B_{X}\left(\varepsilon^{\prime}\right)} d_{X}\left(x_{k}, x_{*}\right) .
$$

As the periodic coder-controller corresponding to $\varepsilon^{\prime}$ discussed immediately after (29) is clearly an element of $\mathcal{F}\left(\varepsilon, \varepsilon^{\prime}, \theta\right)$, we must have $\zeta<\varepsilon^{\prime}$. To see that in fact $\zeta=0$, suppose that $\zeta>0$. By (32), $\forall \phi>0 \exists k^{\prime} \in \mathbb{N}$ and $\left(S^{\prime}, \gamma^{\prime}, \delta^{\prime}\right) \in \mathcal{F}\left(\varepsilon, \varepsilon^{\prime}, \theta\right)$ which achieves

$$
\sup _{x_{0} \in B_{X}\left(\varepsilon^{\prime}\right)} d_{X}\left(x_{k}, x_{*}\right) \leq \zeta+\phi \quad \forall k \geq k^{\prime} .
$$

However, as discussed after (29) there is a periodic coder-controller $\left(S^{\zeta}, \gamma^{\zeta}, \delta^{\zeta}\right)$ which sends an open set $L \supset B_{X}(\zeta)$ of initial states into a closed set $K^{\prime} \subset \operatorname{int} B_{X}(\zeta)$ at all positive times, using inputs in $B_{V}(\varepsilon)$ and with average data rate satisfying (30). It is straightforward to show, using openness and compactness, that there exist $a) \xi \in\left(\zeta, \varepsilon^{\prime}\right)$ such that $L \supset B_{X}(\xi) \supset B_{X}(\zeta)$, and $b) \zeta^{\prime}<\zeta$ such that $K^{\prime} \subseteq B_{X}\left(\zeta^{\prime}\right) \subset B_{X}(\zeta)$. Thus, choose $\phi \leq \xi-\zeta$ in (33), apply $\left(S^{\prime}, \gamma^{\prime}, \delta^{\prime}\right) \in \mathcal{F}\left(\varepsilon, \varepsilon^{\prime}, \theta\right)$, and then switch to the periodic coder-controller $\left(S^{\zeta}, \gamma^{\zeta}, \delta^{\zeta}\right)$ at a suitably large time $k=k^{\prime}$, when $x_{k^{\prime}}$ lies inside $B_{X}(\xi)$. We then have a new finitely switched periodic coder-controller $(S, \gamma, \delta) \in \mathcal{F}\left(\varepsilon, \varepsilon^{\prime}, \theta\right)$ which guarantees that for any $x_{0} \in$ $B_{X}\left(\varepsilon^{\prime}\right), d_{X}\left(x_{k}, x_{*}\right) \leq \zeta^{\prime}<\zeta, \forall k>k^{\prime}$. This contradicts the definition of $\zeta$ as the infimum (32) so we conclude that $\zeta=0$.

By (33), we have thus shown that $\forall \varepsilon>0$ there exists $\varrho_{1}>0$ such that $\forall \theta, \phi>0, \varepsilon^{\prime} \in\left(0, \varrho_{1}\right]$, there is a coder-controller $\in \mathcal{F}\left(\varepsilon, \varepsilon^{\prime}, \theta\right)$ which takes the initial state ball $B_{X}\left(\varepsilon^{\prime}\right)$ into $B_{X}(\phi)$ at all sufficiently large times $k \geq k^{\prime}+1$, with controls $u_{k} \in B_{V}(\varepsilon)$ and the state remaining in some compact ball lying strictly inside $B_{X}\left(\varepsilon^{\prime}\right)$ at all times. Furthermore, as the average data rate of each periodic "section" satisfies (30), so does the overall data rate.

It is now straightforward to construct a coder-controller which achieves local uniform asymptotic stability in the sense (26). Let $\left\{\varepsilon_{i}\right\}_{i=0}^{\infty}$ be any sequence of input ball radii tending monotonically to zero and $\left\{\varepsilon_{i}^{\prime}\right\}_{i=0}^{\infty}$ a sequence of corresponding initial state ball radii, as per the definition of local strong invariance in assumption [C]. As each $\varepsilon_{i}^{\prime}>0$ can be as small as pleased, let $\varepsilon_{i}^{\prime}$ tend monotonically to zero as well. Set $\phi_{i}=\varepsilon_{i+1}^{\prime}$ and let $k_{i}^{\prime}$ be the time it takes for the corresponding coder-controller $\left(S^{i}, \gamma^{i}, \delta^{i}\right) \in \mathcal{F}\left(\varepsilon_{i}, \varepsilon_{i}^{\prime}, \theta\right)$ to take the state ball $B_{X}\left(\varepsilon_{i}^{\prime}\right)$ into $B_{X}\left(\varepsilon_{i+1}^{\prime}\right)$. Define $t_{i} \triangleq \sum_{l=0}^{i-1} k_{l}^{\prime}, \forall l \in \mathbb{N}$, with $t_{0} \triangleq 0$. Construct an (infinitely switched periodic) coder-controller $(S, \gamma, \delta)$ by applying $\left(S^{i}, \gamma^{i}, \delta^{i}\right)$ during times $k \in\left[t_{i}, \ldots, t_{i+1}-1\right], \forall i \in \mathbb{Z}_{+}$. Then during each such interval the controls and states are respectively confined to balls $B_{V}\left(\varepsilon_{i}\right)$ and $B_{X}\left(\varepsilon_{i}^{\prime}\right)$, respectively, with radii tending monotonically to zero. Furthermore, by the strong invariance property of the finitely switched periodic coder-controllers used to construct it, the state is confined to a compact set in the interior of $B_{X}\left(\varepsilon_{0}^{\prime}\right)=B_{X}\left(\varepsilon^{\prime}\right)$. Finally, as each periodic section has average data rate satisfying (30), the asymptotic average data rate of $(S, \gamma, \delta)$ also satisfies (30).

\section{LOCAL UNIFORM ASYMPTOTIC STABILITY IN EUCLIDEAN SPACE}

In this section, we place the plant (1) and its inputs in Euclidean spaces, and turn our attention to deriving an explicit formula for the LTFE of the plant at a fixed point. To do so, we exploit Theorem 2, which states that the LTFE is precisely equal to the infimum feedback data rate for LUAS in the sense (26). We then use local real Jordan forms and volume partitioning arguments to derive an expression for the infimum data rate, and hence the LTFE. ${ }^{1}$

First, we define certain conventions used in this section. Sequences $\left\{a_{j}\right\}_{j=0}^{k}$ are denoted $\tilde{a}_{k}$ and $\|\cdot\|$ represents either the Euclidean norm on a vector space or the matrix norm induced by it. Matrices and vectors are written in boldface. Lebesgue measure is denoted $\lambda$, the $n \times n$ identity matrix is denoted by $\mathbf{I}_{n}$, the $m \times n 0$ matrix by $\mathbf{0}_{m \times n}$, the $m \times n$ matrix $\left[\mathbf{I}_{m} \mathbf{0}_{m \times(n-m)}\right]$ by $\mathbf{I}_{m \times n}$, and the spectrum of a matrix is represented as $\sigma(\cdot)$, with multiple eigenvalues permitted.

Let the state space $X=\mathbb{R}^{n}$, the input space $V=\mathbb{R}^{m}$, and assume that A)-C) from the previous section still hold, with the following additional assumptions.

D) (Continuous differentiability) $F(\cdot, \cdot)$ is differentiable once with continuous first-order partial derivatives.

E) The pair $(\mathbf{A}, \mathbf{B})$ is controllable, where $\mathbf{A}$ and $\mathbf{B}$ are the Jacobians of $F$ w.r.t. state and control respectively at $\left(\mathbf{x}_{*}, \mathbf{u}_{*}\right)$.

The main result of this section follows.

Theorem 3: Let assumptions A)-E) hold on the plant $F(1)$. Then, the local topological feedback entropy (25) of the plant at the fixed point $\left(\mathbf{x}_{*}, \mathbf{u}_{*}\right)$ is given by

$$
h_{*}\left(F, \mathbf{x}_{*}, \mathbf{u}_{*}\right)=\sum_{\eta \in \sigma(\mathbf{A}):|\eta| \geq 1} \log _{2}|\eta|
$$

where $\mathbf{A}$ is the Jacobian of $F$ with respect to state, evaluated at the set-point, and $\sigma(\mathbf{A})$ is the spectrum of $\mathbf{A}$.

This theorem states that the rate at which a plant generates information at a fixed point is determined by the unstable local open-loop dynamics at the desired set-point. The RHS is simply the base- 2 rate at which a volume in the local unstable subspace increases over time, so LTFE here measures the rate at which initial state uncertainty volumes are increased by the action of the plant dynamics. Note that for the case of a linear system, this result is consistent with the work in [11], [3], and [12] on infimum data rates for stabilizability in various other senses.

The remainder of this section is devoted to proving Theorem 3. From Theorem 2, we can do so by first showing that for local uniform asymptotic stabilizability in the sense (26) with some coder-controller (13)-(14), the the data rate $R(12)$ must satisfy

$$
R \geq \sum_{\eta \in \sigma(\mathbf{A}):|\eta| \geq 1} \log _{2}|\eta|
$$

${ }^{1}$ The material in this section was presented in preliminary form in [30]. 
This is done in the following section, using the volume interpretation mentioned above. We then demonstrate the tightness of the data rate lower bound in Section V-C, by constructing a coder-controller that achieves local uniform asymptotic stability at a data rate arbitrarily close to it.

\section{A. Necessity}

The intuition we use to prove the necessity of (35) is that the open-loop growth in unstable subspace uncertainty volume near the set-point must be counteracted by a reduction in volume due to the coding partitions. Similar ideas have been employed for linear plants [11], [3], [12]. However, the nonlinearity of the plant here complicates matters and necessitates rather different technical tools.

Suppose that local uniform asymptotic stability has been achieved by some coder-controller $(S, \gamma, \delta)$ with an initial state ball $B_{X}\left(l_{0}\right)$. Recall that $\mathbf{A}$ is the Jacobian of the dynamical map w.r.t. the state at the set-point

$$
\left.\mathbf{A} \triangleq \frac{\partial F}{\partial \mathbf{x}}\right|_{\left(\mathbf{x}_{*}, \mathbf{u}_{*}\right)} \in \mathbb{R}^{n \times n}
$$

and let $\mathbf{T} \in \mathbb{R}^{n \times n}$ be a real similarity transform such that

$$
\mathbf{J} \triangleq \mathbf{T A T}^{-1} \in \mathbb{R}^{n \times n}
$$

is a real Jordan form; see, e.g., [31] for details. Briefly, $\mathbf{J}$ has a block-diagonal structure with each block possessing either one real or two complex conjugate eigenvalues, not counting repeats. In terms of plant dynamics, $\mathbf{z}_{k} \triangleq \mathbf{T} \mathbf{x}_{k}$ can then be interpreted as a vector of modes with decoupled open-loop dynamics near the target point.

Define $\mathbf{z}_{k}^{\mathrm{u}} \in \mathbb{R}^{d}$ to be the vector of those modes governed by eigenvalues of $\mathbf{J}$ not less than 1 in magnitude. Assuming without loss of generality that the blocks of $\mathbf{J}$ are ordered according to descending eigenvalue magnitudes

$$
\mathbf{z}_{k}^{\mathrm{u}}=\mathbf{I}_{d \times n} \mathbf{x}_{k}, \quad \text { with } \mathbf{z}_{*}^{\mathrm{u}} \triangleq \mathbf{I}_{d \times n} \mathbf{x}_{*} .
$$

It then trivially follows that $\left\|\mathbf{T}\left(\mathbf{x}_{k}-\mathbf{x}_{*}\right)\right\|=\left\|\mathbf{z}_{k}-\mathbf{z}_{*}\right\| \geq$ $\left\|\mathbf{z}_{k}^{\mathrm{u}}-\mathbf{z}_{*}^{\mathrm{u}}\right\|$, so that $\mathbf{z}_{k}^{\mathrm{u}} \rightarrow \mathbf{z}_{*}^{\mathrm{u}}$ uniformly over $\mathbf{x}_{0} \in B_{X}\left(l_{0}\right)$.

Next, for any symbol sequence $\tilde{c}_{k-1} \in \widetilde{S}_{k-1}$ define the $l o$ cally unstable uncertainty set

$$
\begin{aligned}
I_{k}\left(\tilde{c}_{k-1}\right) & :=\left\{\mathbf{z}^{\mathrm{u}} \in \mathbb{R}^{d}: \exists \mathbf{x}_{0} \in B_{X}\left(l_{0}\right)\right. \text { s.t. } \\
\tilde{s}_{k-1} & \left.=\tilde{c}_{k-1} \& \mathbf{z}^{\mathrm{u}}=\mathbf{z}_{k}^{\mathrm{u}}\right\}
\end{aligned}
$$

i.e., the set of all possible points that $\mathbf{z}_{k}^{u}$ can take given the symbol sequence $\tilde{s}_{k-1}=\tilde{c}_{k-1}$. Then, define the maximum locally unstable uncertainty volume

$$
v_{k}:=\max _{\tilde{c}_{k-1}} \lambda\left\{I_{k}\left(\tilde{c}_{k-2}\right)\right\}
$$

Now, if $r$ denotes the supremum distance of points in a measurable set $H \subset \mathbb{R}^{d}$ from $\mathbf{z}_{*}^{\mathrm{u}}$, then $H$ is wholly contained in the ball of radius $r$ centred at $\mathbf{z}_{*}^{\mathrm{u}}$. Hence

$$
\lambda\{H\} \leq \beta r^{d}=\beta \sup _{\mathbf{z}^{\mathrm{u}} \in H}\left\|\mathbf{z}-\mathbf{z}_{*}^{\mathrm{u}}\right\|^{d} \quad \forall H \subset \mathbb{R}^{d}
$$

where $\beta$ is the $d$-dimensional sphere constant. Thus

$$
\begin{aligned}
l_{k} & :=\sup _{\mathbf{x}_{0} \in B_{X}\left(l_{0}\right)}\left\{\left\|\mathbf{z}_{k}-\mathbf{z}_{*}\right\|,\left\|\mathbf{u}_{k}-\mathbf{u}_{*}\right\|\right\} \\
& \geq \sup _{\mathbf{x}_{0} \in B_{X}\left(l_{0}\right)}\left\|\mathbf{z}_{k}^{\mathrm{u}}-\mathbf{z}_{*}^{\mathrm{u}}\right\| \\
& =\max _{\tilde{c}_{k-1}} \sup _{\mathbf{x}_{0} \in B_{X}\left(l_{0}\right), \tilde{s}_{k-1}=\tilde{c}_{k-1}}\left\|\mathbf{z}_{k}^{\mathrm{u}}-\mathbf{z}_{*}^{\mathrm{u}}\right\| \\
& \geq \max _{\tilde{c}_{k-1}}\left(\frac{\lambda\left\{\mathbf{z}_{k}^{\mathrm{u}}-\mathbf{z}_{*}^{\mathrm{u}}: \mathbf{x}_{0} \in B_{X}\left(l_{0}\right), \tilde{s}_{k-1}=\tilde{c}_{k-1}\right\}}{\beta}\right)^{1 / d} \\
& =\max _{\tilde{c}_{k-1}}\left(\frac{\lambda\left\{\mathbf{z}_{k}^{\mathrm{u}}: \mathbf{x}_{0} \in B_{X}\left(l_{0}\right), \tilde{s}_{k-1}=\tilde{c}_{k-1}\right\}}{\beta}\right)^{1 / d} \\
& =\beta^{-1 / d} \max _{\tilde{c}_{k-1}} \lambda\left\{I_{k}\left(\tilde{c}_{k-1}\right)\right\} \equiv \beta^{-1 / d} v_{k}^{1 / d}
\end{aligned}
$$

i.e., $v_{k} \rightarrow 0$ as well. Equality (42) is a consequence of the invariance of Lebesgue measure to constant translations, while the equality in (41) follows from the fact that, with the codercontroller fixed, the same $\mathbf{x}_{0}$ cannot yield two different symbol sequences, i.e., the regions $\left\{\mathbf{x}_{0} \in \mathbb{R}^{n}: \mathbf{x}_{0} \in B_{X}\left(l_{0}\right), \tilde{s}_{k-1}=\right.$ $\left.\tilde{c}_{k-1}\right\}_{\tilde{c}_{k-1} \in \tilde{S}_{k-1}}$ must be disjoint and exhaustive.

A recursive lower bound for the worst-case volume $v_{k}$ will now be derived. We have

$$
\begin{aligned}
v_{k+1} & =\max _{\tilde{c}_{k-1}} \lambda\left\{\mathbf{z}_{k+1}^{\mathrm{u}}: \tilde{s}_{k}=\tilde{c}_{k}\right\} \\
& =\max _{\tilde{c}_{k}} \lambda\left\{G\left(\mathbf{z}_{k}^{\mathrm{u}}, \mathbf{z}_{k}^{\mathrm{s}}, \mathbf{u}_{k}\right): \tilde{s}_{k}=\tilde{c}_{k}\right\}
\end{aligned}
$$

where for convenience, the locally stable components of $\mathbf{T} \mathbf{x}_{k}$ are denoted $\mathbf{z}_{k}^{\mathrm{s}} \in \mathbb{R}^{n-d}$ and $G\left(\mathbf{z}^{\mathbf{u}}, \mathbf{z}^{\mathbf{s}}, \mathbf{u}\right) \triangleq \mathbf{I}_{d \times n} \mathbf{T} F(\mathbf{x}, \mathbf{u})$. The next step is to replace the nonlinear function $G$ with its local linearization. As $F$ has continuous first-order derivatives, we have that $\forall \mathbf{z}, \mathbf{u}$ s.t. $\left\|\mathbf{z}-\mathbf{z}_{*}\right\|,\left\|\mathbf{u}-\mathbf{u}_{*}\right\| \leq l$

$G\left(\mathbf{z}^{\mathrm{u}}, \mathbf{z}^{\mathrm{s}}, \mathbf{u}\right)=\mathbf{z}_{*}^{\mathrm{u}}+\mathbf{J}^{\mathrm{u}}\left(\mathbf{z}^{\mathrm{u}}-\mathbf{z}_{*}^{\mathrm{u}}\right)+\mathbf{I}_{d \times n} \mathbf{T B}\left(\mathbf{u}-\mathbf{u}_{*}\right)+\mathrm{o}(l)$

where the $o(l)$ term $\rightarrow 0$ uniformly over $\mathbf{z}, \mathbf{u}$, and $\mathbf{J}^{\mathbf{u}}:=$ $\mathbf{I}_{d \times n} \mathbf{T J T}^{-1} \mathbf{I}_{n \times d} \in \mathbb{R}^{d \times d}$, the real Jordan form governing the locally unstable subspace. From this it can established that $\exists \varepsilon(l) \rightarrow 0$ s.t. for any measurable $H \subset\left\{(\mathbf{z}, \mathbf{u}) \in \mathbb{R}^{n} \times \mathbb{R}^{m}:\right.$ $\left.\left\|\mathbf{z}-\mathbf{z}_{*}\right\|,\left\|\mathbf{u}-\mathbf{u}_{*}\right\| \leq l\right\}$

$$
\begin{aligned}
& \lambda\left\{G\left(\mathbf{z}^{\mathrm{u}}, \mathbf{z}^{\mathrm{s}}, \mathbf{u}\right):(\mathbf{z}, \mathbf{u}) \in H\right\} \geq \\
& \quad[1-\varepsilon(l)] \lambda\left\{\mathbf{z}_{*}^{\mathrm{u}}+\mathbf{J}^{\mathrm{u}}\left(\mathbf{z}^{\mathrm{u}}-\mathbf{z}_{*}^{\mathrm{u}}\right)+\mathbf{I}_{d \times n} \mathbf{T B u}:(\mathbf{z}, \mathbf{u}) \in H\right\} .
\end{aligned}
$$

Substituting this into (44) with $l=l_{k}, \mathbf{z}_{k}^{\mathrm{u}}=\mathbf{z}^{\mathrm{u}}, \mathbf{z}_{k}{ }^{\mathrm{s}}=\mathbf{z}^{\mathrm{s}}, \mathbf{u}_{k}=$ $\mathbf{u}$, and $\left\{\left(\mathbf{z}_{k}, \mathbf{u}_{k}\right): \tilde{s}_{k}=\tilde{c}_{k}\right\}=H$, and writing $\varepsilon\left(l_{k}\right) \equiv \varepsilon_{k}$

$$
\begin{aligned}
v_{k+1} \geq & \left(1-\varepsilon_{k}\right) \max _{\tilde{c}_{k}} \lambda\left\{\mathbf{z}_{*}^{\mathrm{u}}+\mathbf{J}^{\mathrm{u}}\left(\mathbf{z}_{k}^{\mathrm{u}}-\mathbf{z}_{*}^{\mathrm{u}}\right)\right. \\
& \left.+\mathbf{I}_{d \times n} \mathbf{T B} \delta_{k}\left(\tilde{s}_{k}\right): \tilde{s}_{k}=\tilde{c}_{k}\right\} \\
= & \left(1-\varepsilon_{k}\right) \max _{\tilde{c}_{k}} \lambda\left\{\mathbf{z}_{*}^{\mathrm{u}}+\mathbf{J}^{\mathrm{u}}\left(\mathbf{z}_{k}^{\mathrm{u}}-\mathbf{z}_{*}^{\mathrm{u}}\right)\right. \\
& \left.+\mathbf{I}_{d \times n} \mathbf{T B} \delta_{k}\left(\tilde{c}_{k}\right): \tilde{s}_{k}=\tilde{c}_{k}\right\} \\
= & \left(1-\varepsilon_{k}\right) \max _{\tilde{c}_{k}} \lambda\left\{\mathbf{J}^{\mathrm{u}}\left(\mathbf{z}_{k}^{\mathrm{u}}-\mathbf{z}_{*}^{\mathrm{u}}\right): \tilde{s}_{k}=\tilde{c}_{k}\right\} \\
= & \left(1-\varepsilon_{k}\right) \max _{\tilde{c}_{k}}\left|\operatorname{det} \mathbf{J}^{\mathrm{u}}\right| \lambda\left\{\mathbf{z}_{k}^{\mathrm{u}}: \tilde{s}_{k}=\tilde{c}_{k}\right\} \\
\equiv & \left(1-\varepsilon_{k}\right)\left|\operatorname{det} \mathbf{J}^{\mathrm{u}}\right| \\
& \times \max _{\tilde{c}_{k-1}}\left\{\max _{c_{k}} \lambda\left\{\mathbf{z}_{k}^{\mathrm{u}} s_{k}=c_{k}, \tilde{s}_{k-1}=\tilde{c}_{k-1}\right\}\right\}
\end{aligned}
$$


where (45) follows from the translation-invariance of Lebesgue measure and (46) describes the effect of an invertible linear transformation on volume.

The trivial decomposition (47) leads to an observation that is the heart of the necessity argument developed here. The uncertainty sets $\left\{\mathbf{z}_{k}^{\mathrm{u}}: s_{k}=c_{k}, \tilde{s}_{k-1}=\tilde{c}_{k-1}\right\}$ are not necessarily disjoint as the single symbol $c_{k}$ runs over its possible values. However, since $s_{k}$ is a well-defined function of the initial state and previous symbols, their union must cover the set $\left\{\mathbf{z}_{k}^{\mathrm{u}} \mid \tilde{s}_{k-1}=\tilde{c}_{k-1}\right\}$, i.e.,

$$
\left\{\mathbf{z}_{k}^{\mathrm{u}}: \tilde{s}_{k-1}=\tilde{c}_{k-1}\right\}=\bigcup_{c_{k} \in S_{k}}\left\{\mathbf{z}_{k}^{\mathrm{u}}: s_{k}=c_{k}, \tilde{s}_{k-1}=\tilde{c}_{k-1}\right\} .
$$

Hence

$$
\begin{aligned}
\lambda\left\{\mathbf{z}_{k}^{\mathrm{u}}: \tilde{s}_{k-1}=\tilde{c}_{k-1}\right\} & \leq \sum_{c_{k} \in S_{k}} \lambda\left\{\mathbf{z}_{k}^{\mathrm{u}}: s_{k}=c_{k}, \tilde{s}_{k-1}=\tilde{c}_{k-1}\right\} \\
& \leq \mu_{k} \max _{c_{k} \in S_{k}} \lambda\left\{\mathbf{z}_{k}^{\mathrm{u}}: s_{k}=c_{k}, \tilde{s}_{k-1}=\tilde{c}_{k-1}\right\} .
\end{aligned}
$$

Substituting this into (47)

$$
\begin{aligned}
v_{k+1} & \geq\left(1-\varepsilon_{k}\right)\left|\operatorname{det} \mathbf{J}^{\mathrm{u}}\right| \max _{\tilde{c}_{k-1}} \frac{\lambda\left\{\mathbf{z}_{k}^{\mathrm{u}}: \tilde{s}_{k-1}=\tilde{c}_{k-1}\right\}}{\mu_{k}} \\
& =\frac{\left(1-\varepsilon_{k}\right)\left|\operatorname{det} \mathbf{J}^{\mathrm{u}}\right|}{\mu_{k}} v_{k} \geq v_{0} \prod_{j=0}^{k} \frac{\left(1-\varepsilon_{j}\right)\left|\operatorname{det} \mathbf{J}^{\mathrm{u}}\right|}{\mu_{j}}
\end{aligned}
$$

by repeating the recursion $k$ times. As $v_{0}>0$ and $v_{k} \rightarrow 0$, for all sufficiently large $k$ we must have

$$
\prod_{j=0}^{k} \mu_{j} \geq \prod_{j=0}^{k}\left(1-\varepsilon_{j}\right)\left|\operatorname{det} \mathbf{J}^{\mathrm{u}}\right|
$$

Hence

$$
\begin{aligned}
\frac{1}{k+1} \sum_{j=0}^{k} \log _{2} \mu_{j} \geq & \log _{2}\left|\operatorname{det} \mathbf{J}^{\mathrm{u}}\right| \\
& +\frac{1}{k+1} \sum_{j=0}^{k} \log _{2}\left(1-\varepsilon_{j}\right) . \\
\Rightarrow R:= & \liminf _{k \rightarrow \infty} \frac{1}{k+1} \sum_{j=0}^{k} \log _{2} \mu_{j} \\
\geq & \log _{2}\left|\operatorname{det} \mathbf{J}^{\mathrm{u}}\right| \\
& +\liminf _{k \rightarrow \infty} \frac{1}{k+1} \sum_{j=0}^{k} \log _{2}\left(1-\varepsilon_{j}\right) \\
= & \log _{2}\left|\operatorname{det} \mathbf{J}^{\mathrm{u}}\right| \\
= & \log _{2}\left|\prod_{\eta \in \sigma\left(\mathbf{J}^{\mathrm{u}}\right)} \eta\right| \\
= & \sum_{\eta \in \sigma(\mathbf{A}):|\eta| \geq 1} \log _{2}|\eta|
\end{aligned}
$$

where (49) follows since $\varepsilon_{j} \rightarrow 0$. This completes the proof of necessity for (35).

\section{B. Tightness of Bound}

The final step in proving Theorem 3 is to establish that the bound (35) is achievable, i.e., there exist coding and control schemes with asymptotic average data rates arbitrarily close to it that still achieve local uniform asymptotic stability. In order to do, so a specific coder-controller will be constructed and analyzed.

Note that this scheme is not proposed as a practical control law, as issues such as performance, robustness and complexity would then need to be considered. It is intended only to demonstrate that the data rate lower bound (35) can be approached arbitrarily closely from above, making it the infimum data rate for LUAS.

First, recall that the real Jordan form $\mathbf{J}$ of the Jacobian of $F$ w.r.t. state at the set-point has a block diagonal structure

$$
\mathbf{J} \equiv \operatorname{diag}\left(\mathbf{J}_{1}, \ldots, \mathbf{J}_{r}\right) \in \mathbb{R}^{n \times n}
$$

Each block $\mathbf{J}_{i} \in \mathbb{R}^{d_{i} \times d_{i}}$ has either one distinct, real eigenvalue $\eta_{i}$ of multiplicity $m_{i}=d_{i}$, or two distinct, complex conjugate eigenvalues $\eta_{i}, \bar{\eta}_{i}$ of multiplicities $m_{i}=d_{i} / 2$. The components of the transformed state vector $\mathbf{z}_{k}$ corresponding to the block $\mathbf{J}_{i}$ are denoted $\mathbf{z}_{k}^{(i)} \in \mathbb{R}^{d_{i}}$. A further property of these blocks that will be used later is that $\exists \kappa>0$ s.t.

$$
\left\|\mathbf{J}_{i}^{\tau}\right\| \leq \kappa \tau^{d_{i}-1}\left|\eta_{i}\right|^{\tau} \quad \forall N \in \mathbb{N}
$$

This states that powers of a Jordan block grow exponentially according to the magnitude of its eigenvalue, with possibly an extra polynomial factor arising from multiplicity. ${ }^{2}$

Suppose that at time $k=0,\left\|\mathbf{z}_{0}-\mathbf{z}_{*}\right\| \leq$ some known $b_{0}$. Let $R_{0}$ be any number that satisfies (35) and divide times $k \in \mathbb{Z}_{+}$ into epochs $[j \tau, \ldots,(j+1) \tau-1], j \in \mathbb{Z}_{+}$, of some uniform integer duration $\tau$. At time $k=j \tau$, suppose $b_{j}$ is a uniform bound such that

$$
\left\|\mathbf{z}_{j \tau}-\mathbf{z}_{*}\right\| \leq b_{j} \quad \forall\left\|\mathbf{z}_{0}-\mathbf{z}_{*}\right\| \leq b_{0} .
$$

The way in which $\left\{b_{j}\right\}_{j \in \mathbb{N}}$ is generated will be specified later. Overbound this region by an $n$-dimensional cube centred at $\mathbf{z}_{*}$ with sides of length $2 b_{j}$. Then partition this cube by dividing each coordinate axis corresponding to a component of $\mathbf{z}_{j \tau}^{(i)}$ into $M_{i}$ intervals of equal length

$$
M_{i} \triangleq\left\lfloor\left|\xi \eta_{i}\right|^{\tau}\right\rfloor+1, \text { for }\left|\eta_{i}\right| \geq 1, M_{i} \triangleq 1, \text { for }\left|\eta_{i}\right|<1
$$

where $\lfloor\cdot\rfloor$ denotes rounding down and the parameter $\xi>1$ is selected to satisfy

$$
\begin{aligned}
0<d \log _{2} \xi & <R_{0}-\sum_{\left|\eta_{i}\right| \geq 1} d_{i} \log _{2}\left|\eta_{i}\right| \\
& \equiv R_{0}-\sum_{|\eta| \in \sigma(\mathbf{A}):|\eta| \geq 1} \log _{2}|\eta| .
\end{aligned}
$$

${ }^{2}$ To show this, observe that each real Jordan block is similar to a standard Jordan block (or two) and apply an argument from [31, p. 138] 
Note that as the RHS is guaranteed positive, candidates for $\xi$ always exist. The total number of subcuboids thus formed is $\prod_{i=1}^{r} M_{i}^{d_{i}}$, so index them in a predefined way by the integers $0, \ldots, \mu_{j \tau}-1$, where

$$
\mu_{j \tau} \triangleq \prod_{i=1}^{r} M_{i}^{d_{i}}
$$

At time $j \tau$, transmit the index $s_{j \tau}$ of the one which contains $\mathbf{z}_{j \tau}$. At remaining times in the epoch $j \tau+1 \leq k \leq(j+1) \tau-1$, set $\mu_{k}=1$, i.e., transmit no information. Clearly, the asymptotic average data rate is

$$
\begin{aligned}
R & =\frac{1}{\tau} \log _{2}\left(\prod_{i=1}^{r} M_{i}^{d_{i}}\right)=\frac{1}{\tau} \sum_{\left|\eta_{i}\right| \geq 1} d_{i} \log _{2}\left(\left\lfloor\left|\xi \eta_{i}\right|^{\tau}\right\rfloor+1\right) \\
& \leq \frac{1}{\tau} \sum_{\left|\eta_{i}\right| \geq 1} d_{i} \log _{2}\left(2\left|\xi \eta_{i}\right|^{\tau}\right) \\
& =\frac{d}{\tau}+d \log _{2} \xi+\sum_{\left|\eta_{i}\right| \geq 1} d_{i} \log _{2}\left|\eta_{i}\right|<R_{0}
\end{aligned}
$$

by (52), for sufficiently large $\tau$.

Now, consider the controller at the other end of the channel, which receives the symbol $s_{j \tau}$ at time $j \tau$. As it also knows the uniform bound $b_{j}$ and the number of intervals $M_{i}, i=1, \ldots, r$, it then knows which subcuboid $\mathbf{z}_{\tau j}$ lies in and uses its centre as an estimate $\mathbf{q}_{j}$. Hence

$$
\begin{aligned}
\left\|\mathbf{z}_{j \tau}^{(i)}-\mathbf{q}_{j}^{(i)}\right\| & =\left(\sum_{h=1}^{d_{i}}\left|z_{\tau j}^{(i, h)}-q_{j}^{(i, h)}\right|^{2}\right)^{1 / 2} \\
& \leq\left[\sum_{h=1}^{d_{i}}\left(\frac{b_{j}}{M_{i}}\right)^{2}\right]^{1 / 2}=\frac{\sqrt{d_{i}}}{M_{i}} b_{j}
\end{aligned}
$$

where the additional superscript $h$ denotes the scalar components of vectors in $\mathbb{R}^{d_{i}}$. It then calculates the next $n$ control signals $\mathbf{u}_{\tau j}, \ldots, \mathbf{u}_{\tau j+n-1}$ by using the controllability of $(\mathbf{A}, \mathbf{B})$, and hence of $(\mathbf{J}, \mathbf{T B})$, to force the linearised system with nominal initial state $\mathbf{q}_{j}$ to the origin in $n$ steps, i.e., by solving

$$
\begin{aligned}
\sum_{k=j \tau+1}^{j \tau+n} \mathbf{J}^{\tau j+n-k} \mathbf{T B}\left(\mathbf{u}_{k}-\mathbf{u}_{*}\right) & \equiv \mathbf{W}\left(\mathbf{y}_{j}-\mathbf{y}_{*}\right) \\
& =-\mathbf{J}^{n} \mathbf{T}\left(\mathbf{q}_{j}-\mathbf{z}_{*}\right)
\end{aligned}
$$

where $\mathbf{W} \triangleq\left[\mathbf{T B} \mathbf{J T B} \cdots \mathbf{J}^{n-1} \mathbf{T B}\right] \in \mathbb{R}^{n \times n m}$ and $\mathbf{y}_{j} \triangleq$ $\left[\begin{array}{ll}\mathbf{u}_{j \tau+n}^{\prime} & \mathbf{u}_{j \tau+n-2}^{\prime} \cdots \mathbf{u}_{j \tau+1}^{\prime}\end{array}\right]^{\prime} \in \mathbb{R}^{n m}$. The remaining control signals in the epoch are set to $\mathbf{u}_{*}$ Note that as the controllability matrix $\mathbf{W}$ has rank $n$, it possesses $n$ linearly independent columns $\in \mathbb{R}^{n}$ and only the corresponding $n$ scalar components of the stacked control vector $\mathbf{y}_{j}$ are needed. If the inverse of the matrix formed by these columns is padded with $n m-n$ null rows, corresponding to the unnecessary components of $\mathbf{y}_{j}$, to form $\mathbf{V} \in \mathbb{R}^{n m \times n}$, then the stacked control may be expressed more explicitly as

$$
\mathbf{y}_{j}-\mathbf{y}_{*}=-\mathbf{V} \mathbf{J}^{n}\left(\mathbf{q}_{j}-\mathbf{z}_{*}\right)
$$

i.e., a linear function of $\mathbf{q}_{j}-\mathbf{z}_{*}$.

The crucial remaining step is to determine how to update the uniform upper bound $b_{j}$ from one epoch to the next. In the following, we will construct a recursion for $b_{j}$ which ensures that it decays exponentially to zero for a sufficiently large but fixed epoch duration $\tau$. As $b_{j} \geq\left\|\mathbf{z}_{j \tau}-\mathbf{z}_{*}\right\|$ by definition, this will effectively complete the proof.

First observe that, as $\mathbf{q}_{j} \in \mathbb{R}^{n}$ lies in a cube of sides $2 b_{j}$ centered at $\mathbf{z}_{*},\left\|\mathbf{q}_{j}-\mathbf{z}_{*}\right\| \leq \sqrt{n} b_{j}$. In addition, by (57) $\exists C=$ $\left\|\mathbf{V J} \mathbf{J}^{n}\right\|$, independent of $\tau$, and $b_{j}$ s.t. $\left\|\mathbf{u}_{k}-\mathbf{u}_{*}\right\| \leq C b_{j}$ for all times $k$ in the $j$ th epoch. Now consider the map $F$ iterated $\tau$ times from some initial state $\mathbf{z}$ and with inputs $\mathbf{v}_{0}, \ldots, \mathbf{v}_{\tau-1}$, denoted $F_{\mathbf{v}_{\tau-1}} F_{\mathbf{v}_{\tau-2}} \cdots F_{\mathbf{v}_{0}}(\mathbf{z})$ for convenience. By the continuous differentiability of $F$, it follows that $\forall\left\|\mathbf{z}-\mathbf{z}_{*}\right\| \leq b$, $\left\|\mathbf{v}_{t}-\mathbf{u}_{*}\right\| \leq C b$, and $t \in[0, \ldots, \tau-1]$

$$
\begin{gathered}
\| F_{\mathbf{v}_{\tau-1}} \cdots F_{\mathbf{v}_{0}}(\mathbf{z})-\mathbf{z}_{*}-\mathbf{J}^{\tau}\left(\mathbf{z}-\mathbf{z}_{*}\right) \\
-\sum_{t=0}^{\tau-1} \mathbf{J}_{i}^{\tau-1-t} \mathbf{T B}\left(\mathbf{v}_{t}-\mathbf{u}_{*}\right) \| \\
=0\{b\} \equiv \zeta(b) b
\end{gathered}
$$

where $\zeta(b)$, which may depend on $\tau$, approaches 0 as $b \rightarrow 0$. Substituting $\mathbf{z}=\mathbf{z}_{j \tau}, \mathbf{v}_{t}=\mathbf{u}_{j \tau+t}$, rearranging and looking at each $i$ th local mode

$$
\begin{aligned}
& \left\|\mathbf{z}_{(j+1) \tau}^{(i)}-\mathbf{z}_{*}^{(i)}\right\| \\
\leq & \zeta\left(b_{j}\right) b_{j}+\| \mathbf{J}_{i}^{\tau}\left(\mathbf{z}_{j \tau}^{(i)}-\mathbf{z}_{*}^{(i)}\right) \\
& +\sum_{k=j \tau}^{(j+1) \tau-1} \mathbf{J}_{i}^{(j+1) \tau-1-k}\left[\mathbf{T B}\left(\mathbf{u}_{k}-\mathbf{u}_{*}\right)\right]^{(i)} \| \\
= & \zeta\left(b_{j}\right) b_{j}+\| \mathbf{J}_{i}^{\tau}\left(\mathbf{z}_{j \tau}^{(i)}-\mathbf{z}_{*}^{(i)}\right) \\
& +\mathbf{J}_{i}^{\tau-n-1} \sum_{k=j \tau+1}^{j \tau+n} \mathbf{J}_{i}^{j \tau+n-k}\left[\mathbf{T B}\left(\mathbf{u}_{k}-\mathbf{u}_{*}\right)\right]^{(i)} \| \\
= & \zeta\left(b_{j}\right) b_{j}+\| \mathbf{J}_{i}^{\tau}\left(\mathbf{z}_{j \tau}^{(i)}-\mathbf{z}_{*}^{(i)}\right) \\
& \left.+\mathbf{J}_{i}^{\tau-n-1}\left[-\mathbf{J}_{i}^{n+1}\left(\mathbf{q}_{j}^{(i)}\right)-\mathbf{z}_{*}^{(i)}\right)\right] \| \\
= & \zeta\left(b_{j}\right) b_{j}+\left\|\mathbf{J}_{i}^{\tau}\left(\mathbf{z}_{j \tau}^{(i)}-\mathbf{q}_{j}^{(i)}\right)\right\| \\
\leq & \zeta\left(b_{j}\right) b_{j}+\left\|\mathbf{J}_{i}^{\tau}\right\|\left\|\mathbf{z}_{j \tau}^{(i)}-\mathbf{q}_{j}^{(i)}\right\| \\
\leq & \zeta\left(b_{j}\right) b_{j}+\kappa \tau^{d_{i}-1}\left|\eta_{i}\right|^{\tau} \frac{\sqrt{d_{i}} b_{j}}{M_{i}} .
\end{aligned}
$$


Hence

$$
\begin{aligned}
\left\|\mathbf{z}_{(j+1) \tau}-\mathbf{z}_{*}\right\| \equiv & \left(\sum_{i=1}^{r}\left\|\mathbf{z}_{(j+1) \tau}^{(i)}-\mathbf{z}_{*}^{(i)}\right\|^{2}\right)^{1 / 2} \\
\leq & \sqrt{r} \kappa \max _{1 \leq i \leq r}\left\{\tau^{d_{i}-1}\left|\eta_{i}\right|^{\tau} \frac{\sqrt{d_{i}}}{M_{i}}\right\} b_{j} \\
& +\sqrt{r} \zeta\left(b_{j}\right) b_{j} \\
\equiv & {\left[\beta(\tau)+\sqrt{r} \zeta\left(b_{j}\right)\right] b_{j}=: b_{j+1} }
\end{aligned}
$$

where (59) follows from (56), (60) from (50) and (55), and (61) from the triangle inequality.

Now, consider the $b_{j}$-independent term $\beta(\tau)$ on the RHS of (62). If $\left|\eta_{i}\right|<1, M_{i}=1$ and $\tau^{d_{i}-1}\left|\eta_{i}\right|^{\tau} \rightarrow 0$ as $\tau \rightarrow \infty$. If $\left|\eta_{i}\right| \geq 1, M_{i} \geq\left|\xi \eta_{i}\right|^{\tau}$ from (51), so

$$
\tau^{d_{i}-1}\left|\eta_{i}\right|^{\tau} \frac{\sqrt{d_{i}}}{M_{i}} \leq \sqrt{d_{i}} \tau^{d_{i}-1} \xi^{-\tau} \rightarrow 0 \text { as } \tau \rightarrow \infty
$$

Hence, $\beta(\tau)$ can be made arbitrarily small, independently of $b_{j}$. Select some value for $\tau$ large enough that $\beta(\tau)<1$. As for any fixed $\tau \zeta(b) \rightarrow 0$, set $b_{0}$ so that $\forall b \leq b_{0}, \beta(\tau)+\sqrt{r} \zeta(b) \leq$ some selected $\chi<1$. It then follows that $b_{1} \leq \chi b_{0}<b_{0}$, and by induction it can be established that $b_{j+1} \leq \chi b_{j}<b_{j}$.

Hence $b_{j} \leq \chi^{j} b_{0} \rightarrow 0$ exponentially. Recall that there is a constant $C>0$ such that $\left\|\mathbf{u}_{k}-\mathbf{u}_{*}\right\| \leq C b_{j}, \forall k \in[j \tau, \ldots,(j+$ 1) $\tau-1]$. The continuity of $F$ and the linear dependence of $\mathbf{u}_{k}-\mathbf{u}_{*}$ on $\mathbf{q}_{j}-\mathbf{z}_{*}(57)$ can similarly be used to show that $\exists D>0$ such that $\left\|\mathbf{x}_{k}-\mathbf{x}_{*}\right\| \leq D b_{j}, \forall k \in[j \tau, \ldots,(j+$ 1) $\tau-1]$. Thus for all $x_{0} \in B_{X}\left(b_{0}\right)$ a ball of sufficiently small radius $b_{0}$ centered at $x_{*}$, the plant has been locally exponentially stabilized in state and control using controls $u_{k} \in B_{V}\left(C b_{0}\right)$. $\square$.

\section{CONCLUSION}

This paper addressed the fundamental question of how to define the intrinsic rate at which a nonlinear plant generates stability information. By using general open cover techniques, the concept of TFE was introduced and proposed as a rigorous measure of the rate at which a plant on a noncompact topological space generates initial state information. The problem of datarate-limited stabilzation was then addressed. It was proven that the infimum feedback data rate to be able to keep the states confined to a compact set is precisely equal to the TFE of the plant on the target set. By taking appropriate limits in a metric space, the notion of local TFE (LTFE) was then defined at fixed points of the plant. It was then shown that local uniform asymptotic stabilizability to a fixed point is possible if and only if the data rate exceeds the plant LTFE at the fixed point. For continuously differentiable plants in Euclidean space, a formula for LTFE as the sum of the base-2 logarithms of the unstable eigenvalues of the Jacobian at the fixed point was then derived. Extensions of these results to nonlinear systems with disturbances are presently being investigated.

\section{REFERENCES}

[1] D. F. Delchamps, "Stabilizing a linear system with quantized state feedback," IEEE Trans. Automat. Contr., vol. 35, pp. 916-924, Aug. 1990.
[2] W. S. Wong and R. W. Brockett, "Systems with finite communication bandwidth constraints II: Stabilization with limited information feedback," IEEE Trans. Automat. Contr., vol. 44, pp. 1049-1053, July 1999.

[3] J. Baillieul, "Feedback designs in information-based control," in Proc. Workshop Stochastic Theory and Control, B. Pasik-Duncan, Ed. New York: Springer-Verlag, Oct. 2001, pp. 35-57.

[4] _ , "Feedback coding for information-based control - Operating near the data-rate limit," in Proc. 41 st IEEE Conf. Dec. Contr., Dec 2002, pp. 3229-36.

[5] N. Elia and S. K. Mitter, "Stabilization of linear systems with limited information," IEEE Trans. Automat. Contr., vol. 46, pp. 1384-1400, Aug. 2001.

[6] R. W. Brockett and D. Liberzon, "Quantized feedback stabilization of linear systems," IEEE Trans. Automat. Contr., vol. 45, pp. 1279-1289, July 2000.

[7] I. R. Petersen and A. V. Savkin, "Multi-rate stabilization of multivariable discrete-time linear systems via a limited capacity communication channel," in Proc. 40th IEEE Conf. Decision Control, 2001, pp. 304-309.

[8] H. Ishii and B. A. Francis, "Quadratic stabilization of sampled-data systems with quantization," Automatica, vol. 39, no. 10, pp. 1793-1800, 2003.

[9] D. Liberzon, "On stabilization of linear systems with limited information," IEEE Trans. Automat. Contr., vol. 48, pp. 304-307, Feb. 2003.

[10] G. N. Nair and R. J. Evans, "Stabilization with data-rate-limited feedback: Tightest attainable bounds," Syst. Control Lett., vol. 41, no. 1, pp. 49-56, Sept. 2000.

[11] S. Tatikonda and S. Mitter, "Control under communication constraints," in Proc. 38th Annu. Allerton Conf. Communications, Control, and Computing, Oct. 2000, pp. 182-190.

[12] G. N. Nair and R. J. Evans, "Exponential stabilisability of finite-dimensional linear systems with limited data rates," Automatica, vol. 39, pp. 585-593, 2003.

[13] J. Hespanha, A. Ortega, and L. Vasudevan, "Toward the control of linear systems with minimum bit-rate," in Proc. 15th Int. Symp. Mathematical Theory Networks Systems, Univ. Notre Dame, Notre Dame, IN, Aug. 2002.

[14] F. Fagnani and S. Zampieri, "Stability analysis and synthesis for scalar linear systems with a quantized feedback," IEEE Trans. Automat. Contr., vol. 48, pp. 1569-1584, Sept. 2003.

[15] G. N. Nair and R. J. Evans, "Stabilizability of stochastic linear systems with finite feedback data rates," SIAM J. Control Optim., vol. 43, no. 2, pp. 413-436, 2004.

[16] C. E. Shannon, "A mathematical theory of communication," Bell Syst. Tech. J., 1948

[17] T. M. Cover and J. A. Thomas, Elements of Information Theory. New York: Wiley, 1991.

[18] J. P. Eckmann and D. Ruelle, "Ergodic theory of chaos and strange attractors," Rev. Modern Phys., vol. 57, no. 3, pp. 617-656, 1985.

[19] R. Adler, A. Konheim, and M. McAndrew, "Topological entropy," Trans. Amer. Math. Soc., vol. 114, pp. 61-85, 1965.

[20] T. Ward. (1994) Entropy of compact group automorphisms. Dept. Math., Univ. East Anglia, U.K.. [Online]. Available: www.mth.uea.ac.uk/ h720/lecture_notes/

[21] R. Bowen, Equilibrium States and Ergodic Theory of Anosov Diffeomorphisms. New York: Springer-Verlag, 1975.

[22] M. Pollicot and M. Yuri, Dynamical Systems and Ergodic Theory. Cambridge, U.K.: Cambridge Univ. Press, 1998.

[23] G. Zang and P. A. Iglesias, "Nonlinear extension of Bode's integral based on an information-theoretic interpretation," Syst. Control Lett., vol. 50, no. 1, pp. 11-19, Sep 2003.

[24] K. Tsumura and J. Maciejowski, "Stabilizability of SISO control systems under constraints of channel capacities," in 42nd IEEE Conf. Decision Control, Maui, HI, 2003, pp. 193-198.

[25] N. Elia, "Control-oriented feedback communication schemes," in 42nd IEEE Conf. Decision Control, Maui, HI, 2003, pp. 3161-3166.

[26] G. Polya and G. Szego, Problems and Theorems in Analysis. New York: Springer-Verlag, 1976, vol. 1.

[27] J. E. Cohen, "Subadditivity, generalized products of random matrices and operations research," SIAM Rev., vol. 30, no. 1, pp. 69-86, 1988.

[28] D. Liberzon, "Stabilizing a nonlinear system with limited information feedback," in Proc. 42nd IEEE Conf. Decision Control, Maui, HI, Dec. 2003, pp. 182-186.

[29] C. de Persis, "A note on stabilization via communication channel in the presence of input constraints," in Proc. 42nd IEEE Conf. Decision Control, Maui, HI, Dec. 2003. 
[30] G. N. Nair, R. J. Evans, I. M. Y. Mareels, and W. Moran, "Feedback data rates for nonlinear systems," in Proc. Eur. Control Conf., Cambridge, U.K., Sept. 2003.

[31] R. A. Horn and C. R. Johnson, Matrix Analysis. Cambridge, U.K.: Cambridge Univ. Press, 1985.

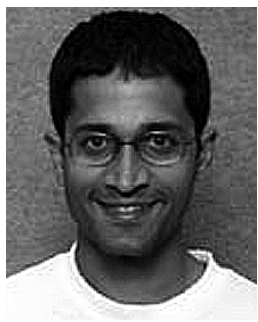

Girish N. Nair (S'97-M'99) was born in Petaling Jaya, Malaysia. He received the B.E. degree (Elec.), the B.Sc. degree (mathematics), and the Ph.D. (electrical engineering), all from the University of Melbourne, Melbourne, Australia, in 1994, 1995, and 2000, respectively.

He is currently a Senior Lecturer in the Department of Electrical and Electronic Engineering, the University of Melbourne, with research interests in the intersection of communications, information theory, and control.

Dr. Nair is the recipient of several prizes, including the Best Theory Paper award at the UKACC International Conference on Control, Cambridge, U.K. (2000) and the LR East Medal in electrical engineering (1994).

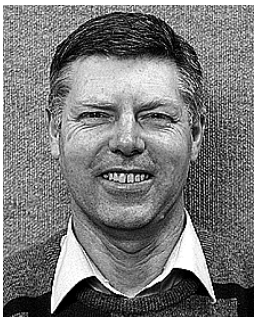

Robin J. Evans (M'80-SM'82-F'02) was born in Melbourne, Australia, in 1947. He received the B.E. degree in electrical engineering from the University of Melbourne, Melbourne, Australia and the Ph.D. degree from the University of Newcastle, Newcastle, Australia, in 1969 and 1975, respectively.

$\mathrm{He}$ worked as a Radar Systems Engineering Officer with the Royal Australian Air Force, followed by postdoctoral studies at the Laboratory for Information and Decision Systems, Massachusetts Institute of Technology, Cambridge, MA, and the Control and Management Department, Cambridge University, Cambridge, U.K. In 1977, he held an academic position at the University of Newcastle, where he was Head of the Department of Electrical and Computer Engineering from 1986 to 1991, and Co-Director of the Centre on Industrial Control Systems from 1988 to 1991. In 1992, he moved to the University of Melbourne, where he was Head of the Department of Electrical and Electronic Engineering until 1996. He is currently Research Leader for the Cooperative Research Centre for Sensor Signal and Information Processing, and Director of the Centre for Networked Decision Systems at the University of Melbourne. His research has ranged across many areas, including theory and applications in industrial control, radar systems, signal processing, and telecommunications.

Prof. Evans is a Fellow of the Australian Academy of Science and the Australian Academy of Technological Sciences and Engineering.

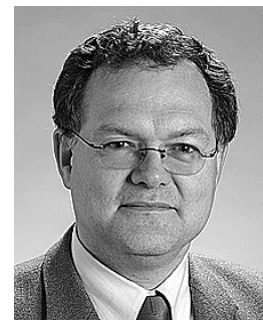

Iven M. Y. Mareels(S'86-M'86-SM'94-F'01) was born in Aalst, Belgium, in 1959. He received the B.S. degree in electromechanical engineering from Gent University, Ghent, Belgium in 1982, and the Ph.D. degree in systems engineering from the Australian National University, Canberra, in 1987.

$\mathrm{He}$ is currently a Professor in the Department of Electrical and Electronic Engineering, the University of Melbourne, Melbourne, Australia, where he holds the Chair of Electrical and Electronic Engineering, a position he took in 1996. Previously, he was a Reade at the Australian National University (1990-1996) and a Lecturer at the University of Newcastle, Newcastle, Australia (1988-1990) and the University of Gent (1986-1988). He has extensive experience in consulting for both industry and government. He has taught a broad range of subjects in both mechanical and electrical engineering curricula. His research interests are in adaptive and learning systems, nonlinear control, and modelling. At present, his research interests are in modelling and controlling of large-scale systems. He is a co-Editorin-Chief of the international journal Systems and Control Letters.

Prof. Mareels was a recipient of the Vice-Chancellor's Award for Excellence in Teaching in 1994. He is a Fellow of the Academy of Technological Sciences and Engineering, Australia, a Member of the Society for Industrial and Applied Mathematics, a Fellow of the Institute of Engineers Australia, Vice-Chair of the Asian Control Professors Association, Chairman of the Education Committee of the latter, and the Chair of the Steering Committee for the Asian Control Conference. He is a Member of the Board of Governors of the IEEE Control Systems Society. He is registered with the Institute of Engineers Australia as a professional engineer.

William Moran (M'95) is a Professorial Fellow in the Department of Electrical and Electronic Engineering at the University of Melbourne, Melbourne, Australia. He also serves as a Consultant to the Australian Department of Defence, through the Defence Science and Technology Organization, and as a Consultant to Prometheus, Inc., Newport, RI. His main areas of interest are in signal processing, particularly with radar applications, waveform design and radar theory, and sensor management. He also works in various areas of mathematics, including harmonic analysis and number theory. 